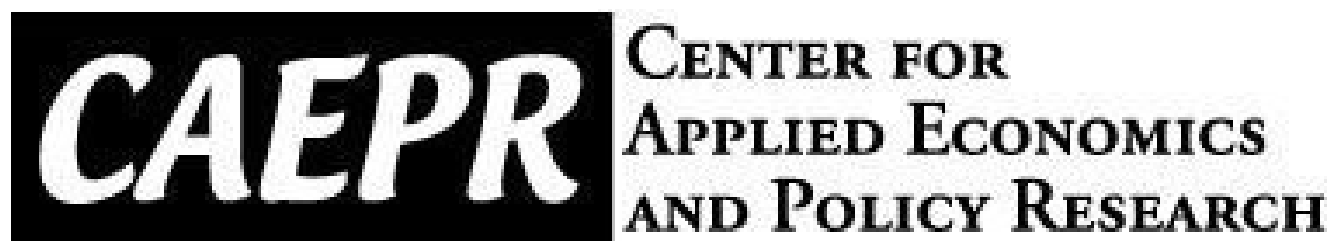

\author{
CAEPR \\ Working Paper \\ \#2018-001
}

\title{
Dynamic Decisions under Subjective Expectations: A Structural Analysis
}

\author{
Yonghong An \\ Texas A\&M \\ University \\ Yingyao Hu \\ Johns Hopkins \\ University \\ Ruli Xiao \\ Indiana University
}

January 2018

This paper can be downloaded without charge from the Social Science Research Network electronic library at https://papers.ssrn.com/abstract_id=3122701

The Center for Applied Economics and Policy Research resides in the Department of Economics at Indiana University Bloomington. CAEPR can be found on the Internet at: http://www.indiana.edu/ caepr. CAEPR can be reached via email at caepr@indiana.edu or via phone at 812-855-4050.

(C)2018 by Yonghong An, Yingyao Hu, and Ruli Xiao. All rights reserved. Short sections of text, not to exceed two paragraphs, may be quoted without explicit permission provided that full credit, including (C) notice, is given to the source. 


\title{
Dynamic Decisions under Subjective Expectations: A Structural Analysis
}

\author{
Yonghong $\mathrm{An}^{*} \quad$ Yingyao $\mathrm{Hu}^{\dagger} \quad$ Ruli Xiao
}

January 2018

\begin{abstract}
This paper studies dynamic discrete choices by relaxing the assumption of rational expectations. That is, agents' subjective expectations about the state transition are unknown and allowed to differ from their objectively estimable counterparts. We show that agents' subjective expectations and preferences can be identified and estimated from the observed conditional choice probabilities in both finite and infinite horizon cases. Our identification of subjective expectations is nonparametric and can be expressed as a closed-form function of the observed conditional choice probabilities. We estimate the model primitives using maximum likelihood estimation and illustrate the good performance of estimators using Monte Carlo experiments. We apply our model to Panel Study of Income Dynamics (PSID) data and analyze women's labor participation. We find systematic differences between agents' subjective expectations about their income transition from those under rational expectations. A counterfactual analysis suggests that women with low and medium incomes would increase the probability of working under rational expectations, and that the probability would decrease for women with high income.
\end{abstract}

Keywords: Dynamic discrete choice models, subjective expectations, rational expectations, nonparametric identification, estimation.

\footnotetext{
*Department of Economics, Texas A\&M University, College Station, TX 77843; email: y.an@tamu.edu.

${ }^{\dagger}$ Department of Economics, Johns Hopkins University, 3100 Wyman Park Dr, Baltimore, MD 21211; email: y.hu@jhu.edu.

${ }_{\ddagger}^{\ddagger}$ Department of Economics, Indiana University, $100 \mathrm{~S}$ Woodlawn Ave, Bloomington, IN 47405; email:rulixiao@iu.edu.
} 


\section{Introduction}

Decision-making under uncertainty, such as occupational and educational choices, and labor participation is prominent in economics. A central problem in this literature is to infer preferences from observed choices. The inference requires a mapping between choices and preferences. To build up the mapping, a general approach is to model agents' choices as the optimal solution to an expected utility maximization problem, where the expected utility is computed using the agents' expectations about choice-specific future outcomes (e.g., a woman's expectations of household income conditional on her labor participation decisions). That is, the observed choices are determined by a combination of preferences and expectations. The information of agents' expectations thus is crucial to infer their preferences through the observed choices.

Unfortunately, researchers typically do not observe agents' expectations in practice, so assumptions usually are imposed. A ubiquitous assumption is that the expectations are rational such that agents' subjective expectations about future outcomes coincide with the ex post realized outcomes. Such an assumption may be problematic, as Manski (1993a) pointed out that the observed choices can be consistent with multiple combinations of expectations and preferences. Moreover, some recent studies have documented systematic discrepancies between subjective and rational expectations by comparing survey data on agents' subjective beliefs with the objective counterparts (see e.g., Heimer, Myrseth, and Schoenle (2017) and Cruces, Perez-Truglia, and Tetaz (2013) among others). Not surprisingly, violation of the rational expectations assumption may induce biased estimation of agents' preferences and misleading counterfactual results. A dominating solution in the literature is to solicit subjective expectations (see a review in Manski (2004)) and to study agents' decisions under the observed expectations (see e.g., Van der Klaauw (2012)). Nevertheless, the availability of such surveyed expectations is very limited. Especially, for some historical datasets, it is impossible to collect agents' subjective expectations.

In the existing literature, little is known about what can be achieved if there are neither solicited subjective expectations nor a known link between the ex post choice-specific outcomes and subjective expectations such as the assumption of rational or myopic expectations. We provide a first positive result in this paper by showing that we can recover agents' preferences as well as subjective expectations if agents' dynamic decisions are observed. Specifically, we consider a standard dynamic discrete choice (DDC) model where agents have subjective beliefs about the law of motion for state variables. The subjective 
expectations are unknown to the econometrician ex ante. This relaxes the rational expectation assumption, which is imposed in this literature to facilitate identification and estimation. We provide conditions for nonparametrically identifying agents' preferences together with their subjective expectations. We analyze both finite and infinite horizon frameworks and provide results for both homogenous and heterogenous beliefs.

We first show that when agents' subjective expectations are homogenous, these expectations can be identified and estimated from the observed conditional choice probabilities (CCPs) in both finite-horizon and infinite-horizon cases. Based on the insight of the under-identification results, e.g., Rust (1994) and Magnac and Thesmar (2002), we address identification of DDC models by assuming that the distribution of agents' unobserved preference shocks and the discount factor are known. Our methodology then identifies agents' subjective probabilities on state transition as a closed-form solution to a set of nonlinear moment conditions that are induced from Bellman equations using the insight in Hotz and Miller (1993). Identifying subjective expectations in the finite-horizon case relies on the variation of agents' CCPs in multiple time periods while those subjective expectations are time-invariant. Our identifying procedure does not require observations at the last period of agents' decisions, although observing such information allows us to achieve identification using data with fewer time periods. In the infinite-horizon scenario, stationarity rules out variation of CCP over time. Our identification relies on the existence of an additional state variable, which enters the utility function linearly. Such an assumption is related but less restrictive than the existence of an exclusion restriction, which is widely used in the literature. However, we require that the law of motion of this additional variable is known to the econometrician. Then we investigate the moment conditions induced from Bellman equations by varying the realizations of this state variable to identify the model.

Our identification strategies also apply to DDC models with agents holding heterogenous subjective expectations and/or preferences. Assuming that agents are classified into finite types and those agents of the same type have homogenous expectations and/or preferences, we prove that the type-specific CCPs can be nonparametrically identified. This step uses the recently developed methodologies in measurement errors, e.g., $\mathrm{Hu}(2008)$. Once the type-specific CCPs are recovered, one can apply the identification results developed for homogenous beliefs to identify the type-specific subjective expectations and/or preferences. Not surprisingly, identification under heterogenous beliefs may require more 
periods of data than is the case with homogenous beliefs.

We propose a maximum likelihood estimator for the model primitives, including agents' preferences and subjective expectations in both finite and infinite horizon cases. Our Monte Carlo experiments show that the proposed estimator performs well with moderate sample sizes, and that performance maintains when the data are generated under rational expectations. Furthermore, we find that imposing rational expectations leads to inconsistent estimation of payoff primitives if the data are generated from subjective expectations that differ from the objective counterparts.

We apply our method to the Panel Study of Income Dynamics (PSID) data to analyze women's labor participation under subjective expectations. To decide whether join the labor force or stay at home, the household need to perceive how would the wife's labor force status affects their future household incomes. We discretize the household income into groups, and refer these groups as low, median, and high. Our estimation results reveal clear discrepancies between subjective expectations about state transitions and their objective counterparts conditional on both working and not working. We also find that agents have an asymmetric judgement about the income transitions: they are overly optimistic about the probabilities of their income becoming higher if current income is low, and overly pessimistic about the probabilities of their income decreasing if current income is high. We further conduct a counterfactual analysis by simulating agents' CCPs under both subjective expectations and rational expectations. Our results suggest heterogenous impacts of subjective expectations on labor participation. Women with low and medium incomes hold rational expectations are more likely to work. In contrast, women with high income are less likely to work if they have rational expectations.

This paper is related to the rapidly growing literature on subjective expectations. Relaxing rational expectations in DDC models, or more generally in decision models, is of both theoretical and empirical importance. Manski (2004) advocates using data on subjective expectations in empirical decision models. In the literature along this line, a great amount of effort has been put into collecting data regarding agents' subjective expectations, and the researchers use these subjective expectations directly to study agents' behaviors under uncertainty. For example, Van der Klaauw and Wolpin (2008) study Social Security and savings using a DDC model where agents' subjective expectations about their own retirement age and longevity and future changes in Social Security policy come from surveys. Zafar (2011, 2013) study schooling choices using surveyed data on students' 
subjective expectations. Wang (2014) uses individuals' subjective longevity expectations to explain adult smokers' smoking decision in a framework of dynamic discrete choices. Acknowledging the scarcity of expectations data, we take a distinctive approach from this literature and focus on understanding agents' decision-making only from their observed choices.

This paper also contributes to a growing literature on identification of dynamic discrete choice models. Rust (1994) provides some non-identification results for the infinite-horizon case. Magnac and Thesmar (2002) further determine the exact degree of underidentification and explore the identifying power of some exclusion restrictions. Kasahara and Shimotsu (2009) and $\mathrm{Hu}$ and Shum (2012) consider identification of DDC models with unobserved heterogeneity/state variables. Fang and Wang (2015) also use exclusion restrictions to identify a DDC model with hyperbolic discounting. Abbring (2010) provides an excellent review on identification of DDC models. In a context of dynamic games, Aguirregabiria and Magesan (2017) consider identification and estimation of players' payoff and belief functions when their beliefs about other players' actions are different from the actual counterparts. Our paper, however, is qualitatively different from these papers in which they assume rational expectations to achieve identification. We are the first to provide rigorous identification results for DDC models allowing that agents have subjective expectations about state transitions. Not surprisingly, our identification and estimation results can be applied to a wide array of empirical studies where agents' subjective expectations are crucial for their decisions but unobserved.

The remainder of this paper is organized as follows. Section 2 presents dynamic discrete choice models with subjective expectations. Sections 3 and 4 propose identification results for the finite and infinite horizon cases, respectively. Section 5 extends identification to the model with heterogeneous beliefs and/or preferences. Section 6 provides an estimator and Monte Carlo evidence. Section 7 studies women's labor participation by applying our method to PSID data. Section 8 concludes and the proofs are presented in the Appendix.

\section{DDC Models with Subjective Expectations}

We consider a single agent DDC model, where each period an agent selects a choice $a_{t}$ from a finite set of actions, $\mathcal{A}=\{1, \cdots, K\}, K \geq 2$, to maximize her expected lifetime 
utility. The state variable that the agent considers at period $t$ has two parts, i.e., $h_{t}=$ $\left(x_{t}, \epsilon_{t}\right)$, where $x_{t}$ is observed by the econometrician, and $\epsilon_{t}$ is the unobserved component, which is a vector of choice-specific shocks, i.e., $\epsilon_{t}=\left(\epsilon_{t}(1), \cdots, \epsilon_{t}(K)\right)$. We assume that the observed state variable $x_{t}$ is discrete and takes values in $\mathcal{X} \equiv\{1, \cdots, J\}, J \geq 2$. At the beginning of period $t, h_{t}$ is revealed and the agent chooses an action $a_{t} \in \mathcal{A}$ and obtains the utility $u\left(x_{t}, a_{t}, \epsilon_{t}\right)$. We use $x$ and $x^{\prime}$ to represent the variable at the current and the following period whenever there is no ambiguity.

After the agent makes the decision $a$, nature determines the realization of the state variable in the next period $\left(h^{\prime}\right)$ according to some random mechanism conditional on the current state $h$ and the agent's decision $a$. This random mechanism usually is assumed to be a Markov process of order one, and we denote it as $f\left(h^{\prime} \mid h, a\right)$. The agent has to form some beliefs about this law of motion in order to take the future into account. The agent's choice $a$ involves intertemporal optimization, thus the belief about the law of motion plays an essential role in the agent's decision making process. Let $s\left(h^{\prime} \mid h, a\right)$ be the agent's subjective beliefs about this law of motion. In the literature of DDC models, a ubiquitous assumption is that the agent has perfect expectations about this law of motion. That is, the subjective expectations are the same as their objective counterparts, i.e., $s\left(h^{\prime} \mid h, a\right)=f\left(h^{\prime} \mid h, a\right)$ for all $h^{\prime}, h$ and $a$. This assumption is imposed to mitigate the complications of identifying and estimating DDC models.

The existing literature provided some rationales and conditions under which rational expectations might hold. For instance, Manski (1993b) argues that perfect expectations of one cohort could be achieved by learning from an earlier cohort. Magnac and Thes$\operatorname{mar}(2002)$ state that the following conditional independence assumption is necessary for agents to have perfect expectations through learning:

Assumption 1 (Conditional Independence) The observed state variable $x^{\prime}$ is drawn independently from $\epsilon$ conditional on $x$ and $a$, i.e., $f\left(x^{\prime} \mid x, a, \epsilon\right)=f\left(x^{\prime} \mid x, a\right)$.

However, Manski (1993b) pointed out that perfect expectations cannot be derived from a learning process if (1) the law of motion changes between the two cohorts due to some macro-level shocks, or (2) the earlier cohort's history cannot be fully observed. Moreover, the recent literature documented violations of perfect expectations by comparing survey data on agents' subjective expectations with the objective counterpart; and such violations often have a great impact on agents' choices. For example, Heimer, Myrseth, and Schoenle 
(2017) show that surveyed mortality beliefs over the life cycle substantially differ from actuarial statistics from the Social Security administration; this discrepancy leads to $30 \%$ under-saving by the young and 15\% more slow drawing down of assets by retirees. Cruces, Perez-Truglia, and Tetaz (2013) also provide evidence of agents' biased perception of the income distribution.

Motivated by these theoretical arguments and the empirical evidence, we consider identification of DDC models without imposing rational expectations on the agent's beliefs about state transition. In what follows, we describe the agent's problem in a general framework with subjective expectations, lay out some traditional assumptions, and characterize the agent's optimization decision.

In each period, the agent's problem is to decide what action maximizes her expected utility, based on her subjective expectations about the future evolution of the payoff state variable. That is,

$$
\max _{a_{t} \in \mathcal{A}} \sum_{\tau=t, t+1, \ldots} \beta^{\tau-t} E\left[u\left(x_{\tau}, a_{\tau}, \epsilon_{\tau}\right) \mid x_{t}, a_{t}, \epsilon_{t}\right]
$$

where $\beta \in[0,1)$ is the discount factor and the expectation is taken using the agent's subjective expectations $s\left(h^{\prime} \mid h, a\right)$. These beliefs are a complete set of conditional probabilities that satisfy the following properties:

Assumption 2 Agents' subjective expectations about the law of motion for the observed state variable satisfy the following conditions:

(a) $\sum_{x^{\prime} \in \mathcal{X}} s\left(x^{\prime} \mid x, a\right)=1$ and $s\left(x^{\prime} \mid x, a\right) \geq 0$ for any $x \in \mathcal{X}$ and $a \in \mathcal{A}$.

(b) $s\left(x^{\prime} \mid x, a\right)$ is time-invariant.

Part (a) of Assumption 2 states some minimum requirements for subjective expectations. That is, the beliefs are valid. Part (b) rules out the possibility that agents update their beliefs about the transition by learning. This is consistent with the recent empirical literature that agents' subjective expectations are often one-time self-reported (see e.g., Wang (2014)).

We further make the following assumptions concerning the unobservable component in the preferences following the literature (e.g., Rust (1987)).

Assumption $3(a) u(x, a, \epsilon)=u(x, a)+\epsilon(a)$ for any $a \in \mathcal{A} ;(b) \epsilon(a)$ are i.i.d. draws from the mean zero type-I extreme value distribution $G(\cdot)$ for all periods and all $a \in \mathcal{A}$. 
The additive separability of agents' utility imposed in Assumption 3 (a) is used widely in the literature. Assuming a known distribution of $\epsilon$ is due to the non-identificatification result of Magnac and Thesmar (2002). The mean zero type-I extreme value distribution is assumed for ease of exposition. Our identification holds for any distribution of $\epsilon$ as long as it is known.

With an additive separable utility function, we can represent the agent's optimal choice $a_{t}$ in period $t$ as

$$
a_{t}=\arg \max _{a \in \mathcal{A}}\left\{u\left(x_{t}, a\right)+\epsilon_{t}(a)+\sum_{\tau=t+1, \ldots} \beta^{\tau-t} E\left[u\left(x_{\tau}, a_{\tau}, \epsilon_{\tau}\right) \mid x_{t}, a\right]\right\}
$$

where the deterministic component of the objective function is defined as the choicespecific value function,

$$
\begin{aligned}
v_{t}(x, a) & \equiv u(x, a)+\sum_{\tau=t+1, \ldots} \beta^{\tau-t} E\left[u\left(x_{\tau}, a_{\tau}, \epsilon_{\tau}\right) \mid x, a\right] \\
& \equiv u(x, a)+\beta \int V_{t}\left(x^{\prime}\right) s\left(x^{\prime} \mid x, a\right) d x^{\prime}
\end{aligned}
$$

where $V_{t}(x)$ is the ex ante value function. The optimization problem can be characterized as a decision rule, which maps the current state and the payoff shocks into an action, denoted as $\delta_{t}:(x, \epsilon) \rightarrow a$. Equivalently,

$$
a=\delta_{t}(x, \epsilon) \quad \Leftrightarrow \quad v_{t}(x, a)+\epsilon_{t}(a)>v_{t}\left(x, a^{\prime}\right)+\epsilon_{t}\left(a^{\prime}\right), \quad \forall a^{\prime} \in \mathcal{A}, a^{\prime} \neq a .
$$

We then define the CCP that the agent chooses each action corresponding to the decision rule $\delta_{t}$. That is,

$$
p_{t}(a \mid x)=\int I\left(a=\delta_{t}(x, \epsilon)\right) d G(\epsilon)
$$

where $I(\cdot)$ is an indicator function.

In a finite horizon case with the ending period denoted as $\widetilde{T}$, the agent can solve for the problem using backward induction. Specifically, in last period $\widetilde{T}$, the choice-specific value function is exactly the payoff function, i.e., $v_{\widetilde{T}}(x, a)=u(x, a)$. As a result, we can 
represent the CCP as follows

$$
p_{\widetilde{T}}(a \mid x)=\int I\left[u(x, a)+\epsilon_{\widetilde{T}}(a)>u\left(x, a^{\prime}\right)+\epsilon_{\widetilde{T}}\left(a^{\prime}\right), \forall a^{\prime} \in \mathcal{A}, a^{\prime} \neq a\right] d G\left(\epsilon_{\widetilde{T}}\right) .
$$

By using backward induction, we can represent the choice-specific value function in period $t$ as the following.

$v_{t}(x, a)=u(x, a)+\beta \int \max _{a^{\prime} \in \mathcal{A}}\left\{v_{t+1}\left(x^{\prime}, a^{\prime}\right)+\epsilon_{t+1}\left(a^{\prime}\right)\right\} s\left(x^{\prime} \mid x, a\right) d G\left(\epsilon_{t+1}\right) d x^{\prime} \quad \forall t<\widetilde{T}$.

Consequently, the agent's CCP induced by the optimal decision rule in period $t$ can be expressed as

$$
p_{t}(a \mid x ; s)=\int I\left[v_{t}(x, a)+\epsilon_{t}(a)>v_{t}\left(x, a^{\prime}\right)+\epsilon\left(a^{\prime}\right)\right] d G\left(\epsilon_{t}\right) \quad \forall t<\widetilde{T}
$$

where $s \equiv\left\{s\left(x^{\prime} \mid x, a\right)\right\}_{a, x, x^{\prime}}$ collects all subjective expectations about the state transition.

In an infinite horizon case, the agent's problem essentially becomes a fixed point problem because stationarity typically is imposed. The decision rule and the corresponding CCPs are consistent in each period, and the choice-specific value functions and the CCPs can be characterized as

$$
\begin{aligned}
p(a \mid x ; s) & =\int I\left[v(x, a)+\epsilon_{t}(a)>v\left(x, a^{\prime}\right)+\epsilon\left(a^{\prime}\right)\right] d G(\epsilon) \\
v(x, a ; s) & =u(x, a)+\beta \int V\left(x^{\prime}\right) s\left(x^{\prime} \mid x, a\right) d x^{\prime}
\end{aligned}
$$

The CCPs then can be represented as a fixed point mapping:

$$
p=\Psi(p ; u, s)
$$

where the mapping $\Psi$ is determined by the distribution of payoff shocks, i.e., $G(\cdot)$, and $p=\{p(a \mid x ; u, s)\}$ collects all CCPs.

Note that in the DDC model introduced above, the state transitions are still governed by the objective probabilities $f\left(x^{\prime} \mid x, a\right)$. Nevertheless, in general the observed choices $\left\{a_{t}\right\}_{t=1,2, \ldots}$ would have different distributions from the case where agents have rational expectations.

Remark. It is worth noting that the subjective expectations about state transitions 
here are qualitatively different from an agent's subjective beliefs about her opponents' behaviors in a dynamic game. An agent's decision rule in our model is a mapping from preference and state transition to a choice, and the mapping remains the same under both subjective and rational expectations about the state transitions. In dynamic games, an agent's strategy is also a mapping from preference, the belief about others' choices, and the state transition, where the belief about others' choices is consistent with the actual choice function at Nash equilibrium. However, this mapping changes when an agent's subjective belief about her opponents' behaviors differs from the actual counterparts. In such a case, agents are boundedly rational, their beliefs are inconsistent, and a Nash equilibrium does not exist. Aguirregabiria and Magesan (2017) consider identification and estimation of dynamic games in such a scenario. Their method that deals with the changed mapping and bounded rationality does not apply to our model. Our model requires an original argument for identification that has not been used in the other contexts, and our method can be extended to dynamic games with Nash equilibrium and agents' having subjective expectations about state transitions.

\section{Identification of Finite Horizon Models}

This section shows that agents' preferences and subjective expectations are uniquely determined by their CCPs. The main idea of identification is to investigate the variation of CCPs across time and to build a relationship between CCPs and subjective expectations. We present an identification procedure for cases with and without observing the last period of agents' decisions. Since the discount factor $\beta$ is not the focus of this paper, we assume that it is known. We refer to Magnac and Thesmar (2002) and Abbring and Daljord (2016) for the identification of the discount factor $\beta$.

\subsection{Identification without the last period}

In this section, we assume that the last period of agents' decisions is not observed in the data, i.e., $t=1,2, \cdots, T$, where $T$ is the last period of data observation but not the final period of agent's decision $\widetilde{T}$, i.e, $T<\widetilde{T}$. Under Assumptions 13 above, the ex ante 
value function at period $t$ can be expressed as

$$
\begin{aligned}
V_{t}(x) & =-\log p_{t}(a=K \mid x)+v_{t}(x, a=K) \\
& \equiv-\log p_{t, K}(x)+v_{t, K}(x)
\end{aligned}
$$

where the choice $K$ can be any choices in $\mathcal{A}$, and we choose the last one for ease of exposition. Given that the state variable $x$ has supports $\{1,2, \ldots, J\}$, we define a vector of $J-1$ independent subjective probabilities as follows:

$$
S_{a}(x)=\left[s\left(x^{\prime}=1 \mid x, a\right), \ldots, s\left(x^{\prime}=J-1 \mid x, a\right)\right], a \in \mathcal{A} .
$$

Similarly, we define

$$
\begin{aligned}
-\log \boldsymbol{p}_{t, K} & =\left[-\log p_{t, K}(x=1), \ldots,-\log p_{t, K}(x=J-1)\right]^{\prime}-\left(-\log p_{t, K}(J)\right) \\
\boldsymbol{v}_{t, K} & =\left[v_{t, K}(x=1), \ldots, v_{t, K}(x=J-1)\right]^{\prime}-v_{t, K}(J) .
\end{aligned}
$$

The choice-specific value function can be expressed as follows:

$$
\begin{aligned}
& v_{t}(x, a)=u(x, a)+\beta \sum_{x^{\prime}} V_{t+1}\left(x^{\prime}\right) s\left(x^{\prime} \mid x, a\right) d x^{\prime} \\
= & u(x, a)+\beta S_{a}(x)\left(-\log \boldsymbol{p}_{t+1, K}+\boldsymbol{v}_{t+1, K}\right)+\beta\left[-\log p_{t+1, K}(J)+v_{t+1, K}(J)\right] .
\end{aligned}
$$

We leave the derivation of equation (6) in the Appendix. We take the difference of the choice-specific value function above between $a=i$ and $a=K$, and apply the results in Hotz and Miller (1993),

$$
\begin{aligned}
\xi_{t, i, K}(x) & \equiv \log \left(\frac{p_{t, i}(x)}{p_{t, K}(x)}\right)=v_{t}(x, a=i)-v_{t}(x, a=K) \\
& =\beta\left[S_{i}(x)-S_{K}(x)\right]\left[-\log \boldsymbol{p}_{t+1, K}+\boldsymbol{v}_{t+1, K}\right]+[u(x, i)-u(x, K)]
\end{aligned}
$$

where $t=1,2, \cdots, T-1$. The difference between the choice probabilities of any two actions, the component in the left-hand-side of the equation above, is time-variant and this variation over time does not rely on the associated utility difference $u(x, i)-u(x, K)$ directly. This property allows us to further eliminate the utility function in the relationship 
between choice-specific value function and CCPs,

$$
\begin{aligned}
\Delta \xi_{t, i, K}(x) & \equiv \log \left(\frac{p_{t, i}(x)}{p_{t, K}(x)}\right)-\log \left(\frac{p_{t-1, i}(x)}{p_{t-1, K}(x)}\right) \\
& =\beta\left[S_{i}(x)-S_{K}(x)\right]\left[-\Delta \log \boldsymbol{p}_{t+1, K}+\Delta \boldsymbol{v}_{t+1, K}\right],
\end{aligned}
$$

where $\Delta \log \boldsymbol{p}_{t+1, K} \equiv \log \boldsymbol{p}_{t+1, K}-\log \boldsymbol{p}_{t, K}$ and $\Delta \boldsymbol{v}_{t+1, K} \equiv \boldsymbol{v}_{t+1, K}-\boldsymbol{v}_{t, K}$. This equation holds for each of $x \in\{1,2, \ldots, J\}$ and any time period $t \in\{1, \cdots, T-1\}$.

Next we stack the equation above for $J-1$ values of $x$ in the matrix form with the following definitions:

$$
\boldsymbol{S}_{a} \equiv\left(\begin{array}{c}
S_{a}(x=1) \\
S_{a}(x=2) \\
\vdots \\
S_{a}(x=J-1)
\end{array}\right) ; \Delta \boldsymbol{\xi}_{t, i, K} \equiv\left[\Delta \xi_{t, i, K}(1), \ldots, \Delta \xi_{t, i, K}(J-1)\right]^{\prime} .
$$

Note that matrix $\boldsymbol{S}_{a}$ has dimensions $(J-1) \times(J-1)$. Then we have a matrix version of equation (8):

$$
\Delta \boldsymbol{\xi}_{t, i, K}=\beta\left[\boldsymbol{S}_{i}-\boldsymbol{S}_{K}\right]\left[-\Delta \log \boldsymbol{p}_{t+1, K}+\Delta \boldsymbol{v}_{t+1, K}\right], \quad \forall t \in\{1, \cdots, T-1\} .
$$

Equation (10) indicates that the observed differences of the CCPs between any two actions over time $\Delta \boldsymbol{\xi}_{t, i, K}$ are induced by two components. One is the difference of the subjective expectations associated with the two actions, which is our identification target. The other one is the difference of the corresponding choice specific value functions, which is also unknown from the data. To identify the subjective expectations, we need to eliminate the value difference $\Delta \boldsymbol{v}_{t+1, K}$ in equation (10). To do that, we explore additional restrictions imposed by the model in the value function, leading to the following connection in the difference of value function over time 1

$$
\Delta \boldsymbol{v}_{t, K}=\beta \widetilde{\boldsymbol{S}}_{K}\left(-\Delta \log \boldsymbol{p}_{t+1, K}+\Delta \boldsymbol{v}_{t+1, K}\right),
$$

\footnotetext{
${ }^{1}$ We provide the detailed derivation of this connection in the Appendix.
} 
where $\widetilde{\boldsymbol{S}}_{K}$ defined as follows is a $(J-1) \times(J-1)$ matrix.

$$
\widetilde{\boldsymbol{S}}_{K} \equiv\left(\begin{array}{c}
S_{K}(1)-S_{K}(J) \\
S_{K}(2)-S_{K}(J) \\
\vdots \\
S_{K}(J-1)-S_{K}(J)
\end{array}\right)
$$

In summary, the choice probabilities are associated with subjective expectations and value functions through equation (10), and the choice-specific value function evolves as in equation (11). By eliminating the value functions from these two equations, we can construct the direct relationship between the observed choice probabilities and the subjective expectations. To proceed, we first impose a rank condition ton the primitive matrices $\boldsymbol{S}_{i}$ and $\boldsymbol{S}_{K}$

Assumption 4 There exists one action $i, i \neq K$ such that the $(J-1) \times(J-1)$ matrix $\boldsymbol{S}_{i}-\boldsymbol{S}_{K}$ is full rank.

Assumption 4 requires that the agent's beliefs about state transition, conditional on choice $i$ relative to choice $K$, have sufficient variations. It guarantees the invertibility of $\boldsymbol{S}_{i}-\boldsymbol{S}_{K}$. A full rank restriction to primitives often is imposed for identification in the literature of structural models. For instance, both Kasahara and Shimotsu (2009) and $\mathrm{Hu}$ and Shum (2012) impose full rank condition in identifying DDC models with unobserved heterogeneity/state variables. Under Assumption 4, we eliminate $\Delta \boldsymbol{v}_{t+1, K}$ in equation (10) to obtain

$$
\beta \widetilde{\boldsymbol{S}}_{K}\left[\boldsymbol{S}_{i}-\boldsymbol{S}_{K}\right]^{-1} \Delta \boldsymbol{\xi}_{t, i, K}-\left[\boldsymbol{S}_{i}-\boldsymbol{S}_{K}\right]^{-1} \Delta \boldsymbol{\xi}_{t-1, i, K}=\beta \Delta \log \boldsymbol{p}_{t, K}, t=3, \cdots, T-1
$$

This equation provides a direct link between the choice probabilities and the subjective expectations through a nonlinear system, which enables us to solve the subjective expectations with a closed-form expression. The nonlinear system above contains $J-1$ equations for a given $t$, and there are $(J-1) \times(J-1)$ and $(J-1) \times(J-1)$ unknown parameters in $\widetilde{\boldsymbol{S}}_{K}$ and $\left[\boldsymbol{S}_{i}-\boldsymbol{S}_{K}\right]^{-1}$, respectively. Suppose that we observe data for $2 J$ consecutive periods, denoted by $t_{1}, \cdots, t_{2 J}$. We assume that the CCPs satisfy 
Assumption 5A Matrix $\Delta \boldsymbol{\xi}_{i, K}$ with dimensions of $(2 J-2) \times(2 J-2)$ is invertible, where

$$
\Delta \boldsymbol{\xi}_{i, K} \equiv\left[\begin{array}{cccc}
\Delta \boldsymbol{\xi}_{t_{1}, i, K} & \Delta \boldsymbol{\xi}_{t_{2}, i, K} & \ldots & \Delta \boldsymbol{\xi}_{t_{2,-}, i, K} \\
\Delta \boldsymbol{\xi}_{t_{1}-1, i, K} & \Delta \boldsymbol{\xi}_{t_{2}-1, i, K} & \ldots & \Delta \boldsymbol{\xi}_{t_{2 J-3}, i, K}
\end{array}\right]
$$

This assumption is imposed on the observed probabilities and therefore is directly testable. Nevertheless, it also rules out the stationary case, where the choice probabilities are timeinvariant. Under this assumption, equation (12) can be rewritten as follows:

$$
\left[\widetilde{\boldsymbol{S}}_{K}\left[\boldsymbol{S}_{i}-\boldsymbol{S}_{K}\right]^{-1}, \quad-\beta^{-1}\left[\boldsymbol{S}_{i}-\boldsymbol{S}_{K}\right]^{-1}\right]=\Delta \log \boldsymbol{p}_{K} \Delta \boldsymbol{\xi}_{i, K}^{-1},
$$

where $\Delta \log \boldsymbol{p}_{K} \equiv\left[\Delta \log \boldsymbol{p}_{t_{1}, K}, \Delta \log \boldsymbol{p}_{t_{2}, K}, \ldots, \Delta \log \boldsymbol{p}_{t_{2 J-2}, K}\right]$. We can solve for $\widetilde{\boldsymbol{S}}_{K}$ and $\boldsymbol{S}_{i}-\boldsymbol{S}_{K}$ from the nonlinear system above. Once $\widetilde{\boldsymbol{S}}_{K}$ is identified, we have obtained $\boldsymbol{S}_{K}(x)-\boldsymbol{S}_{K}(J)$ for all $x \in\{1,2, \cdots, J\}, x \neq J$. In order to fully recover $\boldsymbol{S}_{K}(x)$, we need to pin down $\boldsymbol{S}_{K}(J)$ by the following assumption.

Assumption 6A There exist a state $x \in\{1,2, \cdots, J\}$ under which the agent's subjective expectations about the state transition are known for action $K$.

For ease of exposition, denote the state in Assumption $6 \mathrm{~A}$ as $x=J$, so this assumption indicates that $s\left(x^{\prime} \mid x=J, a=K\right)$ or $\boldsymbol{S}_{K}(J)$ is known. The restriction of known subjective expectations imposed in assumption $6 \mathrm{~A}$ is only required to hold for a certain state and action. For example, the agent might have perfect expectations about the state transition in some extreme states, i.e., $s\left(x^{\prime} \mid J, K\right)=f\left(x^{\prime} \mid J, K\right)$. Assumption $6 \mathrm{~A}$ is supported by some empirical evidence. For example, Heimer, Myrseth, and Schoenle (2017) find from a survey that respondents' subjective survival beliefs equal the actuarial statistics when they turn 68 years old.

The fact that imposing Assumption 6A is necessary for identification comes from the nature of DDC models. Recall that equations (10) and (11) summarize all the structural links in a DDC model with identification power. The two equations suggest that the identification power comes from the variation of CCPs and choice-specific value functions, which depends on the belief difference $\boldsymbol{S}_{i}-\boldsymbol{S}_{K}$ and relative beliefs under choice $K$, $S_{K}(x)-S_{K}(J), x \in \mathcal{J}, x \neq J$. Obviously, different $S_{K}(J)$ can realize both (10) and (11) simultaneously. One possible avenue for relaxing Assumption $6 \mathrm{~A}$ is to explore other 
restrictions provided outside the model. For example, the relationship between subjective expectations and objective transitions.

Under Assumption 6A, we can identify $\boldsymbol{S}_{K}$ and consequently other $\boldsymbol{S}_{i}$. In what follows we show that the subjective expectations associated with other actions $i^{\prime}, i^{\prime} \neq i, K$, can be identified. We augment equation (10) to a matrix equation

$$
\Delta \widetilde{\boldsymbol{\xi}}_{i, K}=\beta\left[\boldsymbol{S}_{i}-\boldsymbol{S}_{K}\right]\left[-\Delta \log \widetilde{\boldsymbol{p}}_{K}+\Delta \widetilde{\boldsymbol{v}}_{K}\right]
$$

where

$$
\begin{aligned}
\Delta \widetilde{\boldsymbol{\xi}}_{i, K} & =\left[\Delta \xi_{\tau_{1}, i, K}, \Delta \xi_{\tau_{2}, i, K}, \ldots, \Delta \xi_{\tau_{J-1}, i, K}\right] \\
\Delta \widetilde{\boldsymbol{v}}_{K} & =\left[\Delta \boldsymbol{v}_{\tau_{1}+1, K}, \Delta \boldsymbol{v}_{\tau_{2}+1, K}, \ldots, \Delta \boldsymbol{v}_{\tau_{J-1}+1, K}\right] \\
\Delta \log \widetilde{\boldsymbol{p}}_{K} & =\left[\Delta \log \boldsymbol{p}_{\tau_{1}+1, K}, \Delta \log \boldsymbol{p}_{\tau_{2}+1, K}, \ldots, \Delta \log \boldsymbol{p}_{\tau_{J-1}+1, K}\right] .
\end{aligned}
$$

The time periods $\tau_{1}, \tau_{2}, \cdots, \tau_{J-1}$ are chosen such that $\Delta \widetilde{\boldsymbol{\xi}}_{i, K}$ is invertible. Note that matrix $\Delta \widetilde{\boldsymbol{\xi}}_{i, K}$ is constructed through reducing the dimension of matrix $\Delta \boldsymbol{\xi}_{i, K}$. Consequently, the existence of such $\left\{\tau_{1}, \tau_{2}, \cdots, \tau_{J-1}\right\}$ is guaranteed by Assumption $5 \mathrm{~A}$ that matrix $\Delta \boldsymbol{\xi}_{i, K}$ is invertible. The invertibility of $\Delta \widetilde{\boldsymbol{\xi}}_{i, K}$, together with Assumption 4 allows us to identify $-\Delta \log \widetilde{\boldsymbol{p}}_{K}+\Delta \widetilde{\boldsymbol{v}}_{K}$ as $\left[\boldsymbol{S}_{i}-\boldsymbol{S}_{K}\right]^{-1} \Delta \widetilde{\boldsymbol{\xi}}_{i, K} / \beta$, which is also invertible. Moreover, equation (14) holds for all other choices $i^{\prime} \neq i, K$, i.e.,

$$
\Delta \widetilde{\boldsymbol{\xi}}_{i^{\prime}, K}=\beta\left[\boldsymbol{S}_{i^{\prime}}-\boldsymbol{S}_{K}\right]\left[-\Delta \log \widetilde{\boldsymbol{p}}_{K}+\Delta \widetilde{\boldsymbol{v}}_{K}\right]
$$

This equation and the identified matrix $-\Delta \log \widetilde{\boldsymbol{p}}_{K}+\Delta \widetilde{\boldsymbol{v}}_{K}$ enable us to identify the subjective expectations associated with choice $i^{\prime}$ as follows.

$$
\begin{aligned}
\boldsymbol{S}_{i^{\prime}} & =\frac{1}{\beta} \Delta \widetilde{\boldsymbol{\xi}}_{i^{\prime}, K}\left[-\Delta \log \widetilde{\boldsymbol{p}}_{K}+\Delta \widetilde{\boldsymbol{v}}_{K}\right]^{-1}+\boldsymbol{S}_{K} \\
& =\Delta \widetilde{\boldsymbol{\xi}}_{i^{\prime}, K} \Delta \widetilde{\boldsymbol{\xi}}_{i, K}^{-1}\left[\boldsymbol{S}_{i}-\boldsymbol{S}_{K}\right]+\boldsymbol{S}_{K}
\end{aligned}
$$

We summarize our identification results as follows:

Theorem 1 Suppose that Assumptions 16 hold. Then the subjective expectations $s\left(x^{\prime} \mid x, a\right)$ for $x, x^{\prime} \in\{1,2, \ldots, J\}$ and $a \in\{1,2, \ldots, K\}$ are identified as a closed-form function of the CCPs $p_{t}(a \mid x), p_{t-1}(a \mid x)$, and $p_{t-2}(a \mid x)$ for $t=t_{1}, t_{2}, \ldots, t_{2 J}$. 
Proof : See the Appendix.

The identification results in Theorem 1 require at least $2 J$ consecutive periods of observations or $2 J-2$ spells of 3 consecutive periods. In empirical applications, we may not have data for $2 J$ periods, especially when the state space, i.e., $J$ is large. We present in the following an alternative identifying strategy that requires only $J+1$ periods of data. Not surprisingly, we have to impose stronger assumption on model primitives than in Theorem 1 .

Assumption 6B There exists an action $a=K$ under which the agent's subjective expectations about state transition for choice $K, s\left(x^{\prime} \mid x, a=K\right)$ are known.

Assumption 6B is stronger than Assumption 6A because it normalizes the whole conditional distribution $s\left(x^{\prime} \mid x, a=K\right)$ for all the values of $x$. Nevertheless, the advantage of such a restriction is that it reduces the number of observed periods required for identification.

To obtain a closed-form solution to subjective expectations, we rewrite the link between CCPs and subjective expectations described in 12 in the following vectorization expression,

$$
\begin{aligned}
\operatorname{vec}\left(\beta \Delta \log \boldsymbol{p}_{K}\right) & =\operatorname{vec}\left(\beta \widetilde{\boldsymbol{S}}_{K}\left[\boldsymbol{S}_{i}-\boldsymbol{S}_{K}\right]^{-1} \Delta \boldsymbol{\xi}_{t, i, K}\right)-\operatorname{vec}\left(\left[\boldsymbol{S}_{i}-\boldsymbol{S}_{K}\right]^{-1} \Delta \boldsymbol{\xi}_{t-1, i, K}\right) \\
& =\left[\left(\Delta \boldsymbol{\xi}_{t, i, K}\right)^{\prime} \otimes\left(\beta \widetilde{\boldsymbol{S}}_{K}\right)\right] \operatorname{vec}\left(\left[\boldsymbol{S}_{i}-\boldsymbol{S}_{K}\right]^{-1}\right)-\left[\left(\Delta \boldsymbol{\xi}_{t-1, i, K}\right)^{\prime} \otimes I\right] \operatorname{vec}\left(\left[\boldsymbol{S}_{i}-\boldsymbol{S}_{K}\right]^{-1}\right) \\
& =\left[\left(\Delta \boldsymbol{\xi}_{t, i, K}\right)^{\prime} \otimes\left(\beta \widetilde{\boldsymbol{S}}_{K}\right)-\left(\Delta \boldsymbol{\xi}_{t-1, i, K}\right)^{\prime} \otimes I\right] \operatorname{vec}\left(\left[\boldsymbol{S}_{i}-\boldsymbol{S}_{K}\right]^{-1}\right)
\end{aligned}
$$

Identification requires the following full rank condition, which again is empirically testable.

Assumption 5B The matrix $\left(\Delta \boldsymbol{\xi}_{t, i, K}\right)^{\prime} \otimes\left(\beta \widetilde{\boldsymbol{S}}_{K}\right)-\left(\Delta \boldsymbol{\xi}_{t-1, i, K}\right)^{\prime} \otimes I$ is invertible.

Consequently, a closed form identification can be represented as

$$
\operatorname{vec}\left(\left[\boldsymbol{S}_{i}-\boldsymbol{S}_{K}\right]^{-1}\right)=\left[\left(\Delta \boldsymbol{\xi}_{t, i, K}\right)^{\prime} \otimes\left(\beta \widetilde{\boldsymbol{S}}_{K}\right)-\left(\Delta \boldsymbol{\xi}_{t-1, i, K}\right)^{\prime} \otimes I\right]^{-1} \operatorname{vec}\left(\beta \Delta \log \boldsymbol{p}_{K}\right)
$$

Similar to the case where $S_{K}(J)$ is known, we can identify $\boldsymbol{S}_{i^{\prime}}$ without imposing any further restrictions after $\boldsymbol{S}_{i}$ is identified. We state the result in the following theorem and omit the proof since it is similar to the proof of Theorem 1 . 
Theorem 2 Suppose that Assumptions 1 4, $5 B$, and $6 B$ hold. Then the subjective expectations $s\left(x^{\prime} \mid x, a\right)$ for $x, x^{\prime} \in\{1,2, \ldots, J\}$ and $a \in\{1,2, \ldots, K-1\}$ are identified as a closed-form function of the CCPs $p_{t}(a \mid x), p_{t-1}(a \mid x)$, and $p_{t-2}(a \mid x)$ for $t=t_{1}, t_{2}, \ldots, t_{J+1}$.

Remark. From the identification procedure above, we can show that the result in Theorem 1 also holds when the discount factor $\beta$ is unknown if we replace Assumption 6A by Assumption $6 \mathrm{~B}$ in Theorem 1. First of all, we can identify $\widetilde{\boldsymbol{S}}_{K}\left[\boldsymbol{S}_{i}-\boldsymbol{S}_{K}\right]^{-1}$ and $\beta^{-1}\left[\boldsymbol{S}_{i}-\boldsymbol{S}_{K}\right]^{-1}$ from equation $(13)$. Under Assumption $6 \mathrm{~B}, \widetilde{\boldsymbol{S}}_{K}$ is known and this allows us to identify $\boldsymbol{S}_{i}-\boldsymbol{S}_{K}$. Consequently, both $\boldsymbol{S}_{i}$ and $\beta$ are identified.

Identification of preferences. After we identify subjective expectations using the results in Theorem 1 or Theorem 2, we proceed to identify agents' utility function $u(x, a)$. As in the existing literature, a normalization is required to identify the preference and is stated in the following.

Assumption 7 The utility of choice $a=K$ is normalized: $u(x, K)=0$ for any $x \in$ $\{1,2, \cdots, J\}$.

Normalization of utility for one alternative is widely used to identify DDC models (see e.g., Blevins (2014), Fang and Wang (2015), and Abbring and Daljord (2016)). The recent literature points out that such normalization is not completely innocuous and may bias counterfactual policy predictions (see e.g., Norets and Tang (2013)). Chou (2015) shows identification of DDC models by relaxing the normalization. Nevertheless, we focus on relaxing rational expectations, thus we maintain this standard assumption.

Since the last period of decision $\widetilde{T}$ is not observed, we cannot use the CCPs in the ending period to identify the preference. Instead we explore the links between the utility function, the observed CCPs, and the identified subjective expectations. Note that the observed CCPs in period $t$ are determined by the choice specific value function at that period $v_{t}(x, a)$, which are time-variant and unknown. This non-stationarity increases the number of unknowns along with the time period. To focus on the preference and reduce the number of unknowns, we represent the difference of CCPs with the choice specific functions in the last period $v_{T}(x, a)$ using the connection of the choice specific value 
function over time 2

$$
\begin{aligned}
& u(x, a)-\beta\left[S_{a}^{\dagger}(x)-S_{K}^{\dagger}(x)\right] \sum_{\tau=t+2}^{T}\left[-\beta S_{K}^{\dagger}(x)\right]^{\tau-t-1}\left(\log \boldsymbol{p}_{\tau, K}^{\dagger}\right) \\
= & \log p_{t, a}(x)-\log p_{t, K}(x)+\beta\left[S_{a}^{\dagger}(x)-S_{K}^{\dagger}(x)\right] \log \boldsymbol{p}_{t+1, K}^{\dagger}+\left(\beta S_{K}^{\dagger}\right)^{T-t-1} \boldsymbol{v}_{T, K}^{\dagger},
\end{aligned}
$$

where $t=1,2, \cdots T-2$, and $x=1,2, \cdots, J . S_{a}^{\dagger}, S_{K}^{\dagger}, \boldsymbol{p}_{t, K}^{\dagger}$ and $\boldsymbol{v}_{t, K}^{\dagger}$ are the counterparts of $S_{a}, S_{K}, \boldsymbol{p}_{t, K}$ and $\boldsymbol{v}_{t, K}$, respectively with the component of $X=J$ included, e.g.,

$$
S_{a}^{\dagger}(x)=\left[S_{a}(x), s\left(x^{\prime}=J \mid x, a\right)\right]=\left[s\left(x^{\prime}=1 \mid x, a\right), \ldots, s\left(x^{\prime}=J \mid x, a\right)\right], \forall a \in \mathcal{A} .
$$

We stack utilities for all the choices and define

$$
\boldsymbol{u}_{a} \equiv\left(\begin{array}{c}
u(x=1, a) \\
u(x=2, a) \\
\vdots \\
u(x=J, a)
\end{array}\right) ; \boldsymbol{S}_{a}^{\dagger} \equiv\left(\begin{array}{c}
S_{a}^{\dagger}(x=1) \\
S_{a}^{\dagger}(x=2) \\
\vdots \\
S_{a}^{\dagger}(x=J)
\end{array}\right), a \in \mathcal{A}, a \neq K
$$

We show in the Appendix that equation (19) implies that for any $t^{\prime} \in\{1,2, \cdots, T-3\}$, $\boldsymbol{u}_{a}$ satisfies the following equation

$$
\begin{aligned}
& {\left[I-\left(\beta \boldsymbol{S}_{K}^{\dagger}\right)^{t^{\prime}}\right] \boldsymbol{u}_{a} } \\
= & \beta\left(\boldsymbol{S}_{a}^{\dagger}-\boldsymbol{S}_{K}^{\dagger}\right)\left[\sum_{\tau=t+2}^{T}\left(-\beta \boldsymbol{S}_{K}^{\dagger}\right)^{\tau-t-1}\left(\log \boldsymbol{p}_{\tau, K}^{\dagger}\right)-\sum_{\tau=t+t^{\prime}+2}^{T}\left(-\beta \boldsymbol{S}_{K}^{\dagger}\right)^{\tau-t-t^{\prime}-1}\left(\log \boldsymbol{p}_{\tau, K}^{\dagger}\right)\right] \\
& +\log \boldsymbol{p}_{t, a}^{\dagger}-\log \boldsymbol{p}_{t, K}^{\dagger}+\beta\left(\boldsymbol{S}_{a}^{\dagger}-\boldsymbol{S}_{K}^{\dagger}\right) \log \boldsymbol{p}_{t+1, K}^{\dagger} \\
& -\left(\beta \boldsymbol{S}_{K}^{\dagger}\right)^{t^{\prime}}\left[\log \boldsymbol{p}_{t+t^{\prime}, a}^{\dagger}-\log \boldsymbol{p}_{t+t^{\prime}, K}^{\dagger}+\beta\left(\boldsymbol{S}_{a}^{\dagger}-\boldsymbol{S}_{K}^{\dagger}\right) \log \boldsymbol{p}_{t+t^{\prime}+1, K}^{\dagger}\right],
\end{aligned}
$$

where the components in the right-hand-side of the equations are either known from the data or are identified from Theorem 1 or Theorem 2, Moreover, we show that the matrix $\left[I-\left(\beta \boldsymbol{S}_{K}^{\dagger}\right)^{t^{\prime}}\right]$ is invertible by construction. Consequently, we provide a closed-form identification expression for the utility function, and summarize the result in the following Corollary.

Corollary 1 Suppose Assumptions 1 hold, agents' utility function $u(x, a)$ is nonparametrically identified.

\footnotetext{
${ }^{2}$ We provide the detailed derivation of equation 190 in the Appendix.
} 
Proof : See Appendix.

\subsection{Identification with the last period}

In this section, we show that the model can be identified using fewer periods of data than are required by Theorems 1,2 if data on the dynamic ending period $\widetilde{T}$ are available. Agents do not need to form beliefs for the future at the ending period. Thus, CCPs on the ending period enables identification of the preference regardless whether agents have subjective or rational expectations. We then do not need to eliminate the utility function from the moment conditions by taking difference over time. Instead we use the identified utility function and the variation of CCPs over time in equation (7) instead of equation (8) to identify the beliefs. We maintain the notation that $t=1,2, \cdots, T$. Here $T$ is the last period of agents' decision, i.e., $T=\widetilde{T}$.

We rewrite equation (7) as follows.

$$
\begin{aligned}
\eta_{t, i, K}(x) & \equiv \xi_{t, i, K}(x)-[u(x, i)-u(x, K)] \\
& =\beta\left[S_{i}(x)-S_{K}(x)\right]\left[-\log \boldsymbol{p}_{t+1, K}+\boldsymbol{v}_{t+1, K}\right], t=1,2, \cdots, T-1
\end{aligned}
$$

where $\eta_{t, i, K}(x)$ is identified because the utility difference $u(x, i)-u(x, K)$ is identified using data of the ending period. Similar to equations 10 and $(11)$, we have the following matrix representation that links the observables to the subjective expectations,

$$
\begin{aligned}
\boldsymbol{\eta}_{t, i, K} & =\beta\left[\boldsymbol{S}_{i}-\boldsymbol{S}_{K}\right]\left[-\log \boldsymbol{p}_{t+1, K}+\boldsymbol{v}_{t+1, K}\right] \\
\boldsymbol{v}_{t, K} & =\beta \widetilde{\boldsymbol{S}}_{K}\left(\log \boldsymbol{p}_{t+1, K}+\boldsymbol{v}_{t+1, K}\right)
\end{aligned}
$$

Under Assumption 4, we have:

$$
\beta \widetilde{\boldsymbol{S}}_{K}\left[\boldsymbol{S}_{i}-\boldsymbol{S}_{K}\right]^{-1} \boldsymbol{\eta}_{t, i, K}-\left[\boldsymbol{S}_{i}-\boldsymbol{S}_{K}\right]^{-1} \boldsymbol{\eta}_{t-1, i, K}=\beta \log \boldsymbol{p}_{t, K}, t=1, \cdots, T
$$

Identification of the subjective expectations can be accomplished with an invertibility assumption stated in the following: 
Assumption 5C Matrix $\boldsymbol{\eta}_{i, K}$ with dimension of $(2 J-2) \times(2 J-2)$ is invertible, where

$$
\boldsymbol{\eta}_{i, K} \equiv\left[\begin{array}{cccc}
\boldsymbol{\eta}_{t_{1}, i, K} & \boldsymbol{\eta}_{t_{2}, i, K} & \ldots & \boldsymbol{\eta}_{t_{2 J-2}, i, K} \\
\boldsymbol{\eta}_{t_{1}-1, i, K} & \boldsymbol{\eta}_{t_{2}-1, i, K} & \ldots & \boldsymbol{\eta}_{t_{2 J-3}, i, K}
\end{array}\right]
$$

Similar to Assumption 5A, Assumption 5C is also testable. We summarize the identification result in the following corollary to Theorem 1 .

Corollary 2 Suppose that Assumptions 14 4, 5C, and 6A hold. Then the subjective expectations $s\left(x^{\prime} \mid x, a\right)$ for $x, x^{\prime} \in\{1,2, \ldots, J\}$ and $a \in\{1,2, \ldots, K\}$ are identified as a closed-form function of the CCPs $p_{t}(a \mid x)$, for $t=t_{1}, t_{2}, \ldots, t_{2 J-2}$.

Corollary 2 shows that $2 J-2$ periods of data (versus $2 J$ periods required in Theorem 1 ) are sufficient for identification if the last period of data are available.

Analogously, if the last period of data are available and Assumption 6B is imposed, i.e., $\boldsymbol{S}_{K}$ is known, we can improve upon Theorem 2 by identifying the model with $J-1$ periods of data. We provide some brief discussions on the identification as the procedure is similar to that of Corollary 22. First of all, utility function can be recovered from the choice in the last period. Using this information and the known subjective expectations $\boldsymbol{S}_{K}$, we can identify the choice-specific value function $\boldsymbol{v}_{t, K}$ for $t=1,2, \cdots, T$ through iteration,

$v_{t}(x, K)=u(x, K)+\beta S_{K}(x)\left(-\log \boldsymbol{p}_{t+1, K}+\boldsymbol{v}_{t+1, K}\right)+\beta\left[-\log p_{t+1, K}(J)+v_{t+1, K}(J)\right]$,

for $t=1, \cdots, T-1$, and the last period choice specific value function is the same as the per-period utility function. To identify the beliefs $\boldsymbol{S}_{i}$, we only need to use the first equation of $(23)$ with $\boldsymbol{S}_{i}$ being the only unknown. We define

$$
\widetilde{\boldsymbol{\eta}}_{i, K}=\left[\boldsymbol{\eta}_{\tau_{1}, i, K}, \boldsymbol{\eta}_{\tau_{2}, i, K}, \ldots, \boldsymbol{\eta}_{\tau_{J-1}, i, K}\right]
$$

which is an observed $(J-1) \times(J-1)$ matrix. A testable full rank condition is necessary for identification.

Assumption 5D The $(J-1) \times(J-1)$ matrix $\widetilde{\boldsymbol{\eta}}_{i, K}$ is invertible.

We summarize the result as a corollary to Theorem 2 : 
Corollary 3 Suppose that Assumptions 1 - 3, and 5D hold. Then the subjective expectations $s\left(x^{\prime} \mid x, a\right)$ for $x, x^{\prime} \in\{1,2, \ldots, J\}$ and $a \in\{1,2, \ldots, K\}$, together with the utility function $u(x, a)$, are identified as a closed-form function of the CCPs $p_{t}(a \mid x)$ for $t=T-J+2, T-J+3, \ldots, T$.

\section{The Infinite Horizon Case}

The identification strategy in Section 3 uses variations in the CCPs across time. Unfortunately, these variations are not available in the infinite horizon case. Thus we explore extra restrictions such as the existence of an additional state variable. We follow the basic setup in Section 2 and maintain Assumptions 1 , 3 in what follows.

Denote the observed state variable to be $(x, w)$, where both are discrete, $x \in\{1,2, \cdots, J\}$ and $w \in\{1,2, \cdots, M\}$. We impose the following assumption on the agents' subjective expectations about the transition of the state variables.

Assumption 8 The agent's subjective beliefs about the transition of the observed state variables $(x, w)$ satisfy

$$
s\left(x^{\prime}, w^{\prime} \mid x, w, a\right)=s\left(x^{\prime} \mid x, a\right) f\left(w^{\prime} \mid w, a\right)
$$

This assumption imposes two restrictions on agents' subjective expectations. First, the two state variables are independent conditional on their previous state. Second, agents' have rational expectation on the transition of state variable $w$. The law of motion for $w$, $f\left(w^{\prime} \mid w, a\right)$ in Assumption 8 is specified to be dependent on action $a$ and our identification in this section will be based on this specification. This nests the law of motion $f\left(w^{\prime} \mid w\right)$, which does not depend on the action, as a special case and the identification results in this section naturally carries through.

The independence of the transitions for the two state variables $x$ and $w$ in Assumption 8 is often assumed in the literature (e.g., see the applications of DDC models reviewed in Aguirregabiria and Mira (2010)). The assumption of known transition of $w$ can be rationalized by the fact that agents often have better understanding of some variables' transition than others'. Especially, when $w$ is a variable at macro level and its transition does not depend on the choice, agents' subjective expectations about its transition can be accurate (see e.g., Manski (2004)). 
We maintain Assumption 6B regarding the subjective beliefs $s\left(x^{\prime} \mid x, a\right)$. That is, all the elements of $s\left(x^{\prime} \mid x, a=K\right)$ are known. Given Assumption 8, we can express the choice-specific value function as

$$
\begin{aligned}
v_{a}(x, w) & =u(x, w, a) \\
& +\beta \sum_{x^{\prime}=1}^{J} \sum_{w^{\prime}=1}^{M}\left[-\log p_{K}\left(x^{\prime}, w^{\prime}\right)+v_{K}\left(x^{\prime}, w^{\prime}\right)\right] s\left(x^{\prime} \mid x, a\right) f\left(w^{\prime} \mid w, a\right) \\
& =u(x, w, a)+\beta \sum_{x^{\prime}=1}^{J}\left[-\log p_{K}^{a}\left(x^{\prime}, w\right)+v_{K}^{a}\left(x^{\prime}, w\right)\right] s\left(x^{\prime} \mid x, a\right),
\end{aligned}
$$

where

$$
\begin{aligned}
\log p_{K}^{a}\left(x^{\prime}, w\right) & \equiv \sum_{w^{\prime}=1}^{M} \log p_{K}\left(x^{\prime}, w^{\prime}\right) f\left(w^{\prime} \mid w, a\right) \\
v_{K}^{a}\left(x^{\prime}, w\right) & \equiv \sum_{w^{\prime}=1}^{M} v_{K}\left(x^{\prime}, w^{\prime}\right) f\left(w^{\prime} \mid w, a\right) .
\end{aligned}
$$

Similar to the finite horizon scenario, the difference between CCPs across actions provide information on the variation of the subjective expectations associated with corresponding actions. Specifically, the mapping between choice-specific value function and CCPs as in Hotz and Miller (1993) leads to the following

$$
\begin{aligned}
\xi_{i, K}(x, w) \equiv & \log \left(\frac{p_{i}(x, w)}{p_{K}(x, w)}\right)=v(x, w, i)-v(x, w, K) \\
= & \beta S_{i}^{x}(x)\left[-\log \boldsymbol{p}_{K}^{i}(w)+\boldsymbol{v}_{K}^{i}(w)\right]-\beta S_{K}^{x}(x)\left[-\log \boldsymbol{p}_{K}^{K}(w)+\boldsymbol{v}_{K}^{K}(w)\right] \\
& +u(x, w, i),
\end{aligned}
$$

where

$$
\begin{aligned}
S_{i}^{x}(x) & =\left[s\left(x^{\prime}=1 \mid x, a\right), \ldots, s\left(x^{\prime}=J \mid x, a\right)\right], \\
\log \boldsymbol{p}_{K}^{i}(w) & =\left[\log p_{K}^{i}(1, w), \ldots, \log p_{K}^{i}(J, w)\right]^{T}, \\
\boldsymbol{v}_{K}^{i}(w) & =\left[v_{K}^{i}(1, w), \ldots, v_{K}^{i}(J, w)\right]^{T}
\end{aligned}
$$

$u(x, w, K)$ is not in equation (27) because of Assumption 7, i.e., $u(x, w, K)=0$. This normalization is necessary due to the non-identification results in the existing literature. Note that in this equation, only CCPs are known, while the utility and the choice-specific value function are yet to be identified. The main idea of identification is to eliminate both the utility and the value function in equation (27). 
We first show that the choice-specific value function $\boldsymbol{v}_{K}$ can be identified using a fixed point relationship when normalization is imposed on action $K$. For choice $a=K$, equation 26 is simplified as

$$
v_{K}(x, w)=\beta \sum_{x^{\prime}=1}^{J} \sum_{w^{\prime}=1}^{M}\left[-\log p_{K}\left(x^{\prime}, w^{\prime}\right)+v_{K}\left(x^{\prime}, w^{\prime}\right)\right] s\left(x^{\prime} \mid x, K\right) f\left(w^{\prime} \mid w, K\right) .
$$

Rewritten in a matrix form,

$$
\boldsymbol{v}_{K}=\beta\left[\boldsymbol{S}_{K}^{x} \otimes \boldsymbol{F}_{K}^{w}\right]\left[-\log \boldsymbol{p}_{K}+\boldsymbol{v}_{K}\right]
$$

where $\boldsymbol{v}_{K}$ is a $J M \times 1$ vector defined as

$$
\boldsymbol{v}_{K} \equiv\left[v_{K}(x=1, w=1), \cdots, v_{K}(x=1, w=M), \cdots, v_{K}(x=J, w=M)\right]^{T} .
$$

The $J M \times 1$ vector of CCPs, $\log \boldsymbol{p}_{K}$, is defined analogously. $\boldsymbol{S}_{K}^{x}$ and $\boldsymbol{F}_{K}^{w}$ are $J \times J$ and $M \times M$ transition matrices, respectively, and are defined as follows.

$$
\boldsymbol{S}_{a}^{x} \equiv\left(\begin{array}{c}
S_{a}^{x}(x=1) \\
S_{a}^{x}(x=2) \\
\vdots \\
S_{a}^{x}(x=J)
\end{array}\right) ; \boldsymbol{F}_{a}^{w} \equiv\left(\begin{array}{c}
F_{a}^{w}(w=1) \\
F_{a}^{w}(w=2) \\
\vdots \\
F_{a}^{w}(w=L)
\end{array}\right)
$$

Equation (28) allows us to identify $\boldsymbol{v}_{K}$ as the following closed-form expression $?^{3}$

$$
\boldsymbol{v}_{K}=\left[I-\beta\left(\boldsymbol{S}_{K}^{x} \otimes \boldsymbol{F}_{K}^{w}\right)\right]^{-1}\left[\beta\left(\boldsymbol{S}_{K}^{x} \otimes \boldsymbol{F}_{K}^{w}\right)\left(-\log \boldsymbol{p}_{K}\right)\right]
$$

Once the value functions for choice $a=K$ are identified, we proceed to provide conditions to eliminate the utility function in equation (27). This is accomplished by varying $w$ in the CCP ratio $\xi_{i, K}(x, w)$ for a certain class of utility function, which is specified in the following assumption:

Assumption 9 The agent's preference is linear in $w, u(x, w, a)=u^{1}(x, a)+u^{2}(x, a) w$ for all choices $a \in \mathcal{A}$ and $a \neq K$.

\footnotetext{
${ }^{3}$ Following the proof of Corollary 1 in Appendix A.2, we can show that the absolute value of any eigenvalue for $\beta\left(\boldsymbol{S}_{K}^{x} \otimes \boldsymbol{F}_{K}^{w}\right)$ is less than 1. Therefore, all the eigenvalues of matrix $I-\beta\left(\boldsymbol{S}_{K}^{x} \otimes \boldsymbol{F}_{K}^{w}\right)$ are nonzero, i.e., it is invertible.
} 
The linearity of the utility function in $w$ specified above nests the commonly used quasilinear utility function $u(x, w, a)=u^{1}(x, a)+w$ as a special case. In the empirical literature of DDC models, linearity of utility function is often assumed, e.g., in Wang (2014) and Fang and Wang (2015).

Let us define an operator $\Delta_{w}^{2}$ as the second order difference of a function of $w, g(w)$, with respect to $w$.

$$
\Delta_{w}^{2} g(m) \equiv[g(w=m)-g(w=m-1)]-[g(w=m+1)-g(w=m)] .
$$

Applying this operator to equation (27),

$$
\begin{aligned}
& \Delta_{w}^{2} \xi_{i, K}(x, w) \\
= & \beta S_{i}^{x}(x)\left[-\Delta_{w}^{2} \log \boldsymbol{p}_{K}^{i}(w)+\Delta_{w}^{2} \boldsymbol{v}_{K}^{i}(w)\right]-\beta S_{K}^{x}(x)\left[-\Delta_{w}^{2} \log \boldsymbol{p}_{K}^{K}(w)+\Delta_{w}^{2} \boldsymbol{v}_{K}^{K}(w)\right] \\
\equiv & \beta S_{i}^{x}(x) \tilde{\boldsymbol{v}}^{i}(w)-\beta S_{K}^{x}(x) \tilde{\boldsymbol{v}}^{K}(w) .
\end{aligned}
$$

For any given $x \in\{1,2, \cdots, J\}$, the equation above contains $J$ unknowns in $S_{i}^{x}(x)$. By varying $w$, we may obtain enough restrictions to solve for $S_{i}^{x}(x)$ under an invertibility condition imposed on the observables. To have enough restrictions, $w$ takes at least two more values than $x$, we consider $w \in\{0,1, \cdots, J+1\}$ and define

$$
\begin{aligned}
\tilde{\boldsymbol{V}}^{i} & =\left[\tilde{\boldsymbol{v}}^{i}(1), \tilde{\boldsymbol{v}}^{i}(2), \ldots, \tilde{\boldsymbol{v}}^{i}(J)\right], \\
\Delta_{w}^{2} \boldsymbol{\xi}_{i, K}(x) & =\left[\Delta_{w}^{2} \xi_{i, K}(x, 1), \Delta_{w}^{2} \xi_{i, K}(x, 2), \ldots, \Delta_{w}^{2} \xi_{i, K}(x, J)\right] .
\end{aligned}
$$

A matrix form of equation (32) can be expressed as

$$
\Delta_{w}^{2} \boldsymbol{\xi}_{i, K}(x)=\beta S_{i}^{x}(x) \tilde{\boldsymbol{V}}^{i}-\beta S_{K}^{x}(x) \tilde{\boldsymbol{V}}^{K} .
$$

Note that $S_{K}^{x}(x)$ is known given Assumption 6B. Thus, the agent's subjective beliefs $S_{i}^{x}(x)$ can be solved from the equation above if the following restriction is imposed.

Assumption 10 For any choice $i \in \mathcal{A} \backslash K$, the $J \times J$ matrix $\widetilde{\boldsymbol{V}}^{i}$ is invertible.

This assumption is directly testable because matrix $\widetilde{\boldsymbol{V}}^{i}$ only contains directly estimable entries. Under assumption 10, we may solve for $\boldsymbol{S}_{i}^{x}(x)$, i.e., $s(\cdot \mid x, a)$ with a closed-form:

$$
S_{i}^{x}(x)=\beta^{-1}\left(\Delta_{w}^{2} \boldsymbol{\xi}_{i, K}(x)+\beta S_{K}^{x}(x) \tilde{\boldsymbol{V}}^{K}\right) \times\left(\tilde{\boldsymbol{V}}^{i}\right)^{-1} .
$$


Given that we have identified the subjective expectations, the utility function $u(x, w, i)$ is also identified from equation (27). We summarize the results as follows:

Theorem 3 Suppose that Assumptions 1, 3, 6B, 7, 10 hold. Then, the subjective beliefs $s\left(x^{\prime} \mid x, a\right)$ for $x, x^{\prime} \in\{1,2, \ldots, J\}$ and $a \in\{1,2, \ldots, K\}$, together with the utility function $u(x, w, a)$, are identified as a closed-form function of the CCPs $p(a \mid x, w)$ and objective state transition $f\left(w^{\prime} \mid w, a\right)$.

Remark 1. In the literature of DDC models, an exclusive variable often is used for identification. For example, in Abbring and Daljord (2016) and Fang and Wang (2015) an exclusive restriction is used to identify the discounting factor. Often, such an exclusive variable $Z$ is assumed to have no impacts on the agent's preference while it does affect the state variable's transition. For example, there are two values of $Z$, denoted as $z_{1}$ and $z_{2}$, such that $u\left(z_{1}, a\right)=u\left(z_{2}, a\right)$ and $F\left(z^{\prime} \mid Z=z_{1}, a\right) \neq F\left(z^{\prime} \mid Z=z_{2}, a\right)$ for any choice $a$. Furthermore, agents have rational expectations about the state transition of $Z$. Assumption 9 nests a special case where $W$ is an exclusive variable by allowing $u^{2}(X, a)$ to be zero. Of course, in our case an additional restriction to $W$ is that its state transition is independent from that of $X$.

Remark 2. If the transition of $w$ does not depend on action, both the conclusion and proof of Theorem 3 still hold. The only change is that some $w$-related terms will be independent of choice, e.g., $\log \boldsymbol{p}_{K}^{i}(w)$ and $\boldsymbol{v}_{K}^{i}(w)$ in equation 27) will be the same for all the choices. The main identification equation (33) will be

$$
\Delta_{w}^{2} \boldsymbol{\xi}_{i, K}(x)=\beta S_{i}^{x}(x) \widetilde{\boldsymbol{V}}-\beta S_{K}^{x}(x) \widetilde{\boldsymbol{V}}
$$

where $\widetilde{\boldsymbol{V}}$ is independent of choice and assumed to be invertible as Assumption 10. Finally,

$$
S_{i}^{x}(x)=\beta^{-1} \Delta_{w}^{2} \boldsymbol{\xi}_{i, K}(x) \times \tilde{\boldsymbol{V}}^{-1}+S_{K}^{x}(x)
$$

\section{An Extension: Heterogeneous Beliefs}

Agents may display heterogeneous preferences and/or beliefs about transition of the same state variable. We show in this section that a DDC model with such heterogeneity can also be identified using the results in previous sections. We focus our discussion on the finite horizon case, and the procedure is readily applicable to the infinite horizon case. 
Suppose agents can be classified into $H \geq 2$ types, and $H$ is known to the econometrician. $\|^{4}$ Let $\tau \in\{1,2, \ldots, H\}$ denote the unobserved type (heterogeneity) such that all agents of the same type have the same subjective expectations and preferences, denoted as $s\left(x^{\prime} \mid x, a, \tau\right)$ and $u(x, a, \tau)$, respectively. Note that we assume an agent's type does not change over time. Similarly, the CCPs for agents of type $\tau$ in period $t$ is $p_{t}(a \mid x, \tau)$. We use an identification methodology for measurement error models to show that the observed joint distribution of state variables and agents' actions uniquely determines the type-specific CCPs $p_{t}(a \mid x, \tau)$ for all $\tau \in\{1,2, \cdots, H\}$, with which we can apply the results of identification in Sections 3 to identify the heterogeneous expectations $s\left(x^{\prime} \mid x, a, \tau\right)$ and the utility functions $u(x, a, \tau)$ associated with type $\tau$.

We start our identification arguments with the following assumptions.

Assumption $11\left\{a_{t}, x_{t}, \tau\right\}$ follows a first-order Markov process.

The first-order Markov property of action and state variables is widely assumed in the literature on DDC models. This assumption trivially holds with the assumption of first order markov process of the state variable and the fact that agents' decision only depends on the current state. Under this assumption, the observed joint distribution is then associated with the unobserved ones as follows:

$$
\begin{aligned}
& \operatorname{Pr}\left(a_{t+l}, \ldots, a_{t+1}, x_{t+1}, a_{t}, x_{t}, a_{t-1}, \ldots, a_{t-l}\right) \\
= & \sum_{\tau=1}^{H} \operatorname{Pr}\left(a_{t+l}, \ldots, a_{t+1} \mid x_{t+1}, \tau\right) \operatorname{Pr}\left(x_{t+1}, a_{t} \mid x_{t}, \tau\right) \operatorname{Pr}\left(\tau, x_{t}, a_{t-1}, \ldots, a_{t-l}\right) .
\end{aligned}
$$

Let $l$ be an integer such that $H \leq K^{l}$, where $K$ and $H$ are numbers of possible realizations of $a_{t}$ and $\tau$, respectively:5 Suppose $h(\cdot)$ is a known function that maps the support of $\left(a_{t+l}, \ldots, a_{t+1}\right), K^{l}$ to that of $\tau$, i.e., $\{1,2, \ldots, H\}$. We define

$$
\begin{aligned}
& a_{t+}=h\left(a_{t+l}, \ldots, a_{t+1}\right), \\
& a_{t-}=h\left(a_{t-1}, \ldots, a_{t-l}\right) .
\end{aligned}
$$

For a fixed pair $\left(x_{t}, x_{t+1}\right)$, we may consider $a_{t+}, a_{t}$, and $a_{t-}$ as three measurements of the unobserved heterogeneity $\tau$ and use the results in $\mathrm{Hu}(2008)$ to identify the objective

\footnotetext{
${ }^{4}$ The number of type $H$ might be inferred from the data, see e.g., Kasahara and Shimotsu (2009). We assume a known $H$ for ease of exposition.

${ }^{5}$ This restriction is satisfied in most of the empirical application where $K=2$ and $J$ is not very large.
} 
$\operatorname{Pr}\left(x_{t+1}, a_{t} \mid x_{t}, \tau\right)$, which leads to identification of the CCPs $p_{t}\left(a_{t} \mid x_{t}, \tau\right) !^{6}$ It is worth noting that we assume that the support of $a_{t+}$ and $a_{t-}$ happens to be the same as that of $\tau$ for simplicity of our identification argument. Our results can be generalized straightforwardly to that case where the support of $a_{t+}$ and $a_{t-}$ is larger than that of $\tau$.

For a given pair $x_{t}$ and $x_{t+1}$ in $\mathcal{X}$, we define a matrix

$$
M_{a_{t+}, x_{t+1}, x_{t}, a_{t-}}=\left[f\left(a_{t+}=i, x_{t+1}, x_{t}, a_{t-}=j\right)\right]_{i, j} .
$$

Assumption 12 For all $\left(x_{t+1}, x_{t}\right) \in \mathcal{X} \times \mathcal{X}$, matrix $M_{a_{t+}, x_{t+1}, x_{t}, a_{t-}}$ has a full rank of $H$.

Note that assumption 12 is imposed on the matrix which can be estimated directly from the data since both actions and states are observed. As a result, this assumption is empirically testable.

The identification strategy in $\mathrm{Hu}$ (2008) requires an eigenvalue-eigenvector decomposition of a matrix from algebra manipulation of some observed matrices. The uniqueness of such a decomposition requires that the eigenvalues are distinctive and can be ordered, which are guaranteed in the following two assumptions, respectively.

Assumption 13 For any given $\left(x_{t+1}, x_{t}\right) \in \mathcal{X} \times \mathcal{X}$, there exists a choice $k \in \mathcal{A}$ such that $\operatorname{Pr}\left(a_{t}=k \mid x_{t+1}, x_{t}, \tau=l\right) \neq \operatorname{Pr}\left(a_{t}=k \mid x_{t+1}, x_{t}, \tau=m\right)$ if $l \neq m$, and $l, m \in\{1,2, \cdots, H\}$.

Note that this assumption is imposed for one action $k$ instead of for all of the possible actions. Moreover, even though we cannot estimate the $\operatorname{Pr}\left(a_{t}=k \mid x_{t+1}, x_{t}, \tau\right)$ directly from the data, this assumption is still empirically testable since it is the eigenvalues of matrix computed from the data.

Assumption 14 For any given $x_{t+1} \in \mathcal{X}$, there exists a known $m \in\{1,2, \cdots, H\}$ such that $\operatorname{Pr}\left(a_{t+}=m \mid x_{t+1}, \tau\right)$ is strictly monotonic in $\tau$.

Recall that $a_{t+}$ is defined as $h\left(a_{t+l}, \ldots, a_{t+1}\right)$ and $h(\cdot)$ can be any function that maps the support $\mathcal{A}^{l}$ to $\{1,2, \cdots, H\}$. Thus we have the flexibility to impose Assumption 14 by choosing any suitable function $h(\cdot)$. In empirical applications, this assumption is often implied by the model. Suppose we consider a dynamic investment problem with choices

\footnotetext{
${ }^{6}$ Similar results can also be found in Kasahara and Shimotsu (2009).
} 
being investing in stocks or not, and the choice crucially depends on an agent's subjective expectations about stock returns. For illustration purpose, assume that agents have homogenous preferences and heterogeneous expectations. The type $\tau$ captures the accuracy of subjective expectations (i.e., whether they are close to the ex post distribution of returns) and takes three values "more accurate", "medium accurate" and "less accurate", denoted as $\tau=1,2$, and 3, respectively. We choose $l=2$ such that the requirement $H \leq K^{l}$ is satisfied. $h(\cdot)$ maps the support of $\left(a_{t+1}, a_{t+2}\right),\{(0,0),(0,1),(1,0),(1,1)\}$ to $\{1,2,3\}$. One such mapping $h(\cdot)$ is

$$
a_{t+}= \begin{cases}1, & \text { if }\left(a_{t+1}, a_{t+2}\right)=(0,0) \\ 2, & \text { if }\left(a_{t+1}, a_{t+2}\right)=(0,1) \text { or }\left(a_{t+1}, a_{t+2}\right)=(1,0), \\ 3, & \text { if }\left(a_{t+1}, a_{t+2}\right)=(1,1) .\end{cases}
$$

A natural monotonicity that satisfies Assumption 14 is that the more accurate the subjective expectations, the higher probability agents choose to invest in both periods of $t+1$ and $t+2$, i.e.,

$$
\operatorname{Pr}\left(a_{t+1}=3 \mid \tau=3\right)>\operatorname{Pr}\left(a_{t+1}=3 \mid \tau=2\right)>\operatorname{Pr}\left(a_{t+1}=3 \mid \tau=1\right) .
$$

We summarize the identification result in the following theorem.

Theorem 4 Suppose Assumptions 11, 12, 13, and 14 hold, then the joint distribution $\operatorname{Pr}\left(a_{t+l}, \ldots, a_{t}, x_{t+1}, x_{t}, a_{t-1}, \ldots, a_{t-l}\right)$ uniquely determines the type-specific CCPs $p_{t}\left(a_{t} \mid x_{t}, \tau\right)$.

Proof: See the Appendix.

Provided that the type-specific CCPs $p_{t}\left(a_{t} \mid x_{t}, \tau\right)$ are identified, we can proceed to identify both utility and subjective expectations for each type of agents using the results in Section 3 and 4. Note that for agents with two different types, they may differ in subjective expectations or preferences or both. The identified utility function and subjective expectations for each type allow us to distinguish those three scenarios by comparison. 


\section{Estimation and Monte Carlo Evidence}

\subsection{Estimation}

Our identification result provides a closed-form solution to agent's subjective expectations in equations (13) or (18) for the finite horizon and equation (35) for the infinite horizon, which imply closed-form estimators. One may follow the identification procedure to estimate the subjective expectations directly. Agent's preference then can be estimated in a second step using the CCP approach based on Hotz and Miller (1993). A multi-step estimation, however, is not efficient. Also, such a closed-form estimator involves the inverse of some observed matrices, so the performance of the estimator would be unstable when these matrices are nearly singular. Thus, we instead propose a maximum likelihood estimator for estimating the subjective expectations and agent's preferences at one step.

Suppose the data include $n$ agents' actions and states in $T+1$ periods, denoted as $\left\{a_{i t}, x_{i t}\right\}_{i t}$, where $i=1, \ldots, n$ and $t=0, \ldots, T$. We denote the parameters in payoff functions, objective transitions, and subjective expectations as $\theta_{u}, \theta_{o}$, and $\theta_{s}$, respectively. Those parameters can be finitely or infinitely dimensional. We first present the likelihood function of the data $\left\{a_{i t}, x_{i t}\right\}_{i t}$.

$L\left(x_{1}, \ldots, x_{T}, a_{1}, \ldots a_{T} ; x_{0}, a_{0}, \theta_{u}, \theta_{s}, \theta_{o}\right)=\prod_{i=1}^{n} \Pi_{t=1}^{T} p_{t}\left(a_{i t} \mid x_{i t}, \theta_{u}, \theta_{s}\right) f\left(x_{i t} \mid x_{i, t-1}, a_{i t-1}, \theta_{o}\right)$,

where $p_{t}\left(a_{i t} \mid x_{i t}, \theta_{u}, \theta_{s}\right)$ is the CCPs in period $t$. The log-likelihood function is additively separable in the following two components:

$$
\log L=\sum_{i=1}^{n} \sum_{t=1}^{T} \log p_{t}\left(a_{i t} \mid x_{i t}, \theta_{u}, \theta_{s}\right)+\sum_{i=1}^{n} \sum_{t=1}^{T} \log f\left(x_{i t} \mid x_{i t-1}, a_{i t-1}, \theta_{o}\right)
$$

As a result, we can estimate the preferences $\theta_{u}$ and the subjective expectations $\theta_{s}$ separately from estimating the objective transition $\theta_{u}$. Specifically, the parameters regarding the objective state transition $\theta_{o}$ can be estimated by maximizing the log-likelihood function $\sum_{i=1}^{n} \sum_{t=1}^{T} \log f\left(x_{i t} \mid x_{i t-1}, a_{i t-1}, \theta_{o}\right)$, while the preferences $\theta_{u}$ and the subjective expectations $\theta_{s}$ can be estimated from the log-likelihood function $\sum_{i=1}^{n} \sum_{t=1}^{T} \log p_{t}\left(a_{i t} \mid x_{i t}, \theta_{u}, \theta_{s}\right)$. We use $\hat{\theta}$ to denote the estimator of the corresponding parameters.

Recall that we cannot non-parametrically identify all subjective expectations. Some normalization has to be imposed such as subjective expectations associated with some action $K$ and/or state $J$ are known. One potential possibility is that agents have rational 
expectations about some state transition so that we can use the objective transition of these states in the estimation. Specifically, we divide the parameters associated with subjective expectations into two parts: $\theta_{s} \equiv\left\{\theta_{s}^{e}, \theta_{s}^{n}\right\}$, where $\theta_{s}^{n}$ and $\theta_{s}^{e}$ are the parts to be normalized and estimated, respectively. $\theta_{s}^{n}$ can be obtained from the estimated objective state transition $\hat{\theta}_{o}$, denoted as $\hat{\theta}_{s}^{n}$. The parameters of preferences and subjective expectations, $\theta \equiv\left\{\theta_{u}, \theta_{s}^{e}\right\}$ can be estimated through the following maximization,

$$
\max _{\theta, \theta_{s}^{e}} \sum_{i=1}^{n} \sum_{t=1}^{T} \log p_{t}\left(a_{i t} \mid x_{i t}, \theta, \hat{\theta}_{s}^{n}\right)
$$

Since the representation of CCPs $p_{t}\left(a_{i t} \mid x_{i t}, \theta, \theta_{s}^{n}\right)$ varies with the horizon of the models, we present the estimators for both finite and infinite scenarios separately.

Finite horizon. For a finite horizon case, we use backward induction to back out CCPs for each period $t$. Specifically, we start in the last period $\widetilde{T}$, the stopping period, in which the choice-specific value function is the same as the per period flow utility. Then we move to the next to last period, $\widetilde{T}-1$. We can continue the procedure until we reach the first period in our dataset. In this process, the iteration of value function and the relationship between value function and CCP are characterized by equations (1) and (2), respectively.

Infinite horizon. In the case of infinite horizon, an individual's decision problem can be described as a fixed-point solution. We solve the CCPs from a fixed-point mapping as developed in equation (3)

$$
p=\Psi\left(p ; \theta_{u}, \theta_{s}\right)
$$

To estimate $\theta$, we adopt a Nested Pseudo Likelihood Algorithm (NPL) as proposed in Aguirregabiria and Mira (2002). To implement the algorithm, we start with an initial guess for the CCPs. At the $\tau$-th $(\tau \geq 1)$ step of iteration, we take the following two steps.

- Step 1: given $p^{\tau}$, we obtain a new pseudo-likelihood estimate of $\theta, \theta^{\tau}$, which satisfies

$$
\theta^{\tau}=\arg \max _{\theta} \sum_{i=1}^{N} \sum_{t=1}^{T} \log p\left(a_{i t} \mid x_{i t} ; \theta, \hat{\theta}_{s}^{n}\right)
$$

where $p\left(a_{i t} \mid x_{i t} ; \theta\right)$ is an element of $p$ satisfying the original mapping $p=\Psi\left(p^{\tau} ; \theta, \hat{\theta}_{s}^{n}\right)$. 
- Step 2: we then update the CCPs using the new estimated structural parameters $\theta^{\tau}$ following the model

$$
p^{\tau+1}=\Psi\left(p^{\tau} ; \theta^{\tau}, \hat{\theta}_{s}^{n}\right)
$$

We iterate the steps above until $p$ and $\theta$ converge. We refer to Kasahara and Shimotsu (2008) for convergence of the estimator generated from the NPL algorithm to the MLE. In particular, the NPL estimator converges to a MLE estimator at a super-linear, but less than quadratic, rate.

The difference between our estimator and that in the existing literature lies in the role of the subjective expectations in the estimation. Specifically, we estimate part of the subjective expectations $\theta_{s}^{e}$ together with the payoff primitives $\theta_{u}$. In contrast, the existing literature assumes that the subjective expectations are the same as the objective state transitions. They estimate the payoff primitives in the second step while $\theta_{s}$ is estimated directly from data in the first step. If individuals do not have rational expectations, the existing approach suffers from mis-specification and may result in biased estimation.

\subsection{Monte Carlo Experiments}

Here we present Monte Carlo results to illustrate the finite sample performance of the proposed MLE estimator for the finite horizon case and the NPL estimator for the infinite horizon case.

We consider a binary choice DDC model in both finite and infinite horizon scenarios. First we set up the payoff primitives, the objective law of motion, and agent's beliefs about transition of the state variable, where the agent may or may not have perfect expectations. Given these primitives, we solve for the optimal CCPs either by backward induction or by contraction mapping, depending on the horizon of the model. We then use the equilibrium CCPs to simulate agent's actions and use the objective transition matrices to simulate states. Next we estimate the the parameters of interest following the estimators proposed in the previous section. In particular, we first estimate the objective transition matrices using the frequency estimator. Then we estimate payoff primitives and subjective expectations together by using MLE estimator and NPL estimator in finite and infinite horizon cases, respectively. We also estimate the payoff primitives by imposing the rational expectation assumption as in the existing literature for purposes of comparison. 
In the finite horizon case, the utility function is specified as follows,

$$
u(a, x)=\left\{\begin{array}{l}
0+\epsilon_{0}, \text { if } a=0, \\
u_{x}+\epsilon_{1}, \text { if } a=1,
\end{array}\right.
$$

where $\epsilon_{0}$ and $\epsilon_{1}$ are drawn from a mean-zero type-I extreme value distribution. We set $J=3$, i.e., the state variable $x$ takes three values, so we have three utility parameters, i.e., $u_{1}=-2, u_{2}=0.4, u_{3}=2.1$. The objective law of motion for the state variable $x$ conditional on the choice $a=0$ and $a=1$, is represented by the following $3 \times 3$ matrices

$$
\boldsymbol{T R}_{0}=\left[\begin{array}{ccc}
0.8 & 0.1 & 0.1 \\
0.2 & 0.6 & 0.2 \\
0.1 & 0.19 & 0.71
\end{array}\right] ; \quad \boldsymbol{T} \boldsymbol{R}_{1}=\left[\begin{array}{ccc}
0.2 & 0.6 & 0.2 \\
0.5 & 0.2 & 0.3 \\
0.2 & 0.3 & 0.5
\end{array}\right]
$$

respectively. Let $\boldsymbol{S}_{0}$ and $\boldsymbol{S}_{1}$ denote agent's subjective expectations on the law of motion for action 0 and 1, respectively. We consider three different scenarios for the expectations: (1) Agents have perfect expectations, i.e., $\boldsymbol{S}_{1}=\boldsymbol{T} \boldsymbol{R}_{1}$ and $\boldsymbol{S}_{0}=\boldsymbol{T} \boldsymbol{R}_{0}$. (2) Agents' subjective expectations about the state transition associated with action $a=1$ are the same as its objective counterpart, i.e., $\boldsymbol{S}_{1}=\boldsymbol{T} \boldsymbol{R}_{1}$; agents' subjective expectations about the station transition associated with action $a=0$ deviates from the objective counterpart and is expressed in the following matrix:

$$
\boldsymbol{S}_{0}=\left[\begin{array}{ccc}
0.9 & 0.05 & 0.05 \\
0.1 & 0.8 & 0.1 \\
0.05 & 0.095 & 0.855
\end{array}\right]
$$

(3) Agent's subjective expectations on the transition of one state $x=J$ associated with action $a=1$ are the same as its objective counterpart, i.e., $\boldsymbol{S}_{1}(3)=\boldsymbol{T} \boldsymbol{R}_{1}(3)$; the beliefs on the remaining law of motion deviate from their counterparts and are expressed as follows:

$$
\boldsymbol{S}_{0}=\left[\begin{array}{ccc}
0.9 & 0.05 & 0.05 \\
0.1 & 0.8 & 0.1 \\
0.05 & 0.095 & 0.855
\end{array}\right], \quad \boldsymbol{S}_{1}=\left[\begin{array}{ccc}
0.6 & 0.3 & 0.1 \\
0.25 & 0.6 & 0.15 \\
0.2 & 0.3 & 0.5
\end{array}\right]
$$

Settings (2) and (3) correspond to Assumptions 6A and 6B, respectively. The identifica- 
tion requires $J+1=4$ and $2 * J=6$ periods of data in setting (2) and (3), respectively. To compare the performance in the two setting, we simulate the data for $2 * J=6$ periods regardless the setting.

For the infinite horizon setting, we also consider a case where agents' choice is binary but there are two state variable $x$ and $w$. Based on the identification conditions, the payoff function is assumed as follows

$$
u(a, x, w)=\left\{\begin{array}{l}
0+\epsilon_{0}, \text { if } a=0 \\
u^{1}(x)+u^{2}(x) w+\epsilon_{1}, \text { if } a=1
\end{array}\right.
$$

where $\epsilon_{0}$ and $\epsilon_{1}$ are drawn from mean-zero type-I extreme value distribution. Both state variables are assumed to be discrete. Specifically, $x \in\left\{x_{1}, x_{2}\right\}$ and $w \in\left\{w_{1}, w_{2}, w_{3}, w_{4}\right\}$. The objective state transition processes for $x$ and $w$ are assumed to be stationary and action-specific. We represent all objective transition matrices in the following

$$
\begin{aligned}
& \boldsymbol{T R}_{0}^{w}=\left[\begin{array}{cccc}
0.6 & 0.2 & 0.2 & 0 \\
0.1 & 0.75 & 0.15 & 0 \\
0.04 & 0.1 & 0.8 & 0.06 \\
0.01 & 0.08 & 0.1 & 0.81
\end{array}\right] ; \quad \boldsymbol{T} \boldsymbol{R}_{1}^{w}=\left[\begin{array}{cccc}
0.7 & 0.1 & 0.15 & 0.05 \\
0.2 & 0.65 & 0.05 & 0.1 \\
0.04 & 0.01 & 0.9 & 0.05 \\
0.02 & 0.18 & 0.1 & 0.7
\end{array}\right] ; \\
& \boldsymbol{T} \boldsymbol{R}_{0}^{x}=\left[\begin{array}{cc}
0.6 & 0.4 \\
0.45 & 0.55
\end{array}\right] ; \\
& \boldsymbol{T R} \boldsymbol{R}_{1}^{x}=\left[\begin{array}{cc}
0.1 & 0.9 \\
0.5 & 0.5
\end{array}\right] .
\end{aligned}
$$

Agents have rational expectations about the transition of $w$ but may have subjective expectations about transition of $x$. Specifically, we consider two settings for Monte Carlo experiments: (1) Agents have rational expectations; that is, the agent's beliefs on the state evolution are the same as the objective counterparts, i.e., $\boldsymbol{S}_{a}^{w}=\boldsymbol{T} \boldsymbol{R}_{a}^{w}, \boldsymbol{S}_{a}^{x}=\boldsymbol{T} \boldsymbol{R}_{a}^{x}$, $a \in\{0,1\}$. (2) Agents' subjective expectations satisfy Assumptions 6A and 8. Specifically, $\boldsymbol{S}_{a}^{w}=\boldsymbol{T} \boldsymbol{R}_{a}^{w}, a \in\{0,1\}$ and $\boldsymbol{S}_{1}^{x}=\boldsymbol{T} \boldsymbol{R}_{1}^{x}$, while $\boldsymbol{S}_{0}^{x} \neq \boldsymbol{T} \boldsymbol{R}_{0}^{x}$. In particular,

$$
\boldsymbol{S}_{0}^{x}=\left[\begin{array}{ll}
0.7 & 0.3 \\
0.3 & 0.7
\end{array}\right]
$$

We again estimate the models in both settings with and without imposing the rational expectation assumption.

With the above frameworks, we estimate the objective state transition in the first step 
using frequency estimator. Based on the objective state transition and the normalization condition, we estimate the payoff primitive and the subjective expectations together.7 The estimations are conducted for every scenarios with sample sizes $n=300,600,1000,2500$ and standard errors are computed using 1000 replications.

We present the results of Monte Carlo experiments for the finite horizon case in Tables 1.4, and for the infinite horizon case in Tables 5.6. Three main messages can be seen. First, the proposed estimator performs well across different settings for moderate sample sizes. The parameters of the utility function are estimated better than those of the subjective expectations. It is worth noting that our estimator is robust to rational expectations; i.e., the estimates track the true parameter values quite closely, even when the data are generated from rational expectations. In such a case, as shown in Tables 1 and 3 , the standard errors of our estimates are larger than in the approach without estimating agents' subjective expectations. Second, failing to account for subjective expectations may lead to significant estimation bias. Specifically, when the data are generated from subjective expectations, the estimated parameters of the utility function from imposing rational expectations are not consistent and are far from the true values. This can be seen in Tables 2 and 4 , where estimates of $u_{1}, u_{2}$ and $u_{3}$ are significantly different from the true parameters, by $27 \%, 40 \%$ and $22 \%$, respectively. The differences persist as the sample size increases from 300 to 2500. Finally, in the finite horizon case, the results in settings (2) and (3) do not differ significantly. This implies that normalization itself does not affect our estimation significantly.

\section{Empirical Illustration: Women's Labor Force Par- ticipation}

In this section, we apply the proposed method to study women's labor force participation. Specifically, we investigate whether households have rational expectation about their income evolution and how imposing rational expectations will affect our understanding of their labor force participation decisions.

\footnotetext{
${ }^{7}$ Note that we check the full rank condition such as Assumptions 4 and $5 \mathrm{~A}$ before we move to the estimation step.
} 


\subsection{Data and model specification}

The PSID is a longitudinal survey consisting of a nationally representative sample of over 18,000 individuals living in 5,000 families in the United States. The original sample was re-interviewed annually starting from 1968 to 1997 and biennially afterward. We only use data collected before 1997, since our identification relies on variation in CCPs in consecutive years. More importantly, the PSID collects data on annual income and female labor force participation for the preceding calendar year.

We construct an annual employment profile for each woman between ages of 39 and 60 , where 60 is assumed to be the end period of labor participation decision process. The distribution of women by years of data is shown in Table 7. The sample consists of 1767 women who are not evenly distributed over the number of years of participation data. Almost $16 \%$ of them have all 17 years of data and more than a half of them have over 15 years of data. Table 8 presents the summary statistic of our sample, where we aggregate the information of those women who are observed at least for six periods (for the purpose of identification, as discussed below). The table indicates a relative large variation of household income. Over half of the women across all years are employed status. On average, the women in our sample have high school eduction and the majority of them are aged between 42 to 55 .

Each household in the sample is assumed to maximize the present value of utility over a known finite horizon by choosing whether or not the wife works in each discrete period $\square^{8}$ This framework fits into a finite horizon scenario because women typically are out of labor force when they reach 60 years old, e.g., see Eckstein and Wolpin (1989). A household's utility function is specified as

$$
u(a, x, \epsilon)=\left\{\begin{array}{l}
0+\epsilon_{0}, \text { if } a=0, \\
u_{x}+\epsilon_{1}, \text { if } a=1,
\end{array}\right.
$$

where $\epsilon_{0}$ and $\epsilon_{1}$ are drawn from a mean-zero type-I extreme value distribution and are assumed to be independent over time, $a$ is a binary variable equals 1 if the wife works during period $t$, and 0 otherwise; $x$ is the household income. The discount factor $\beta$ is set to be 0.95 . We discretize household income into three values $(J=3)$ and label $x=1,2,3$

\footnotetext{
${ }^{8}$ We assume that a woman and her husband jointly decide whether she works or not.
} 
as low, medium, and high income, respectively.

$$
x= \begin{cases}1, & \text { if household income } \leq 17,000 \\ 2, & \text { if } 17,000<\text { household income } \leq 150,000 \\ 3, & \text { if household income }>150,000\end{cases}
$$

We use the setting of Theorem 1, where identification requires at least $2 J=6$ periods of observations. Thus, we exclude women with observations fewer than 6 periods from our sample.

The inter-temporal link for the household comes through income evolution over time. Specifically, household income in the future depends on current household income and the wife's working status. Households hold subjective expectations about the income transition $s\left(x^{\prime} \mid x, a\right)$, and take into account such a transition in their dynamic decisions. We assume that the subjective expectations are homogenous even women in our sample differ in age and education. We believe that homogeneity of subjective expectations is a reasonable first-order approximation because we only focus on women between ages 39 to 60, and arguably they are all experienced enough such that age and eduction could not affect their subjective expectations significantly. The homogeneity assumption is also a response to the relative small sample size. If the sample size is large enough, we can allow dependence of subjective expectations on a rich set of covariates and conduct our analysis conditional on the covariates.

Recall that identification requires that households' beliefs are partly known, as stated in Assumption 6A. In this application, we assume that the belief about future income distribution for households with high incomes and the wife working, $s\left(x^{\prime} \mid x=3, a=1\right)$, is known to be the transition observed in the data. We believe this is a reasonable assumption because the income for a high income household is less uncertain. Moreover, they may not qualify for some social welfare programs as the low income households do, so it is relative easier for them to predict their income if the wife quits her job.

As in Eckstein and Wolpin (1989), we make some key working assumptions in the specification above. First, we ignore the choice of hours of work and we treat labor supply as a discrete work/no-work decision. Second, we ignore husband's labor force participation decisions. Third, we focus on the decision of women after age 39 so as to avoid model fertility decisions. Finally, marriage is taken as exogenously given. 


\subsection{Estimation results}

Our estimation results are presented in Table 9$]^{9}$ The top panel provides estimates of transition matrices and parameters of utility under subjective expectations. For comparison, in the bottom panel we include the estimates after imposing rational expectations, where the objective transitions of household income are estimated using simple frequency estimators. Table 9 reveals clear discrepancies between subjective expectations and rational expectations about state transitions, conditional on both working and not working. For example, conditional on medium income, agents believe that if the wife works then with $55.6 \%$ probability their income will remain to be medium in the next period; with probability of $24.3 \%$ and $20.1 \%$, they would move to the low and high income categories. By contrast, the objective transitions indicate that with probability $92.1 \%$ a household would stay in the medium income category, much higher than the result of $55.6 \%$. It is possible that they will fall into low and high income categories, but the probabilities of $6.9 \%$ and $1.1 \%$ respectively are much lower than what is perceived by agents.

Another important observation from comparing subjective expectations and objective transitions is that agents have an asymmetric judgement about income transitions. For example, using the estimates conditional on not working, agents are overly optimistic about the probabilities of their income transitioning to medium (24.3\% v.s. 6.9\%) and high $(20.1 \%$ v.s. $1.1 \%)$ conditional on current low income and overly pessimistic about the probabilities of their income decreasing to low (45.0\% v.s. $0.7 \%)$ and medium (30.5\% v.s. $23.7 \%$ ) if the current income is high. This is consistent with some survey data suggesting asymmetric beliefs of agents. For example, Heimer, Myrseth, and Schoenle (2017) find discrepancies between the surveyed mortality beliefs and the actuarial statistics from the Social Security administration, and those discrepancies are different across age groups.

Estimated preferences under subjective expectations and rational expectations share a similar pattern: women prefer to work if their households' incomes are medium or high while they prefer not to work if their current income is low. Nevertheless, our estimates indicate that the utility of working is highest for those whose income is medium, while under rational expectations the highest utility is for high income woman.

Based on the estimates in Table 9, we conduct a counterfactual analysis to investigate how the discrepancies in estimated income transitions and households' preferences affect

\footnotetext{
${ }^{9}$ Before estimating the model parameters, we test the rank of the observed matrix $\Delta \boldsymbol{\xi}_{i, K}$ and find that it is full rank, i.e., Assumption $5 \mathrm{~A}$ holds.
} 
women's labor force participation. Specifically, we simulate households' CCPs for the last six periods using the estimates of preference parameters under subjective expectations, and the estimated income transitions under subjective expectations and rational expectations in Table 9. The simulated CCPs and the percentage differences presented in Table 10 suggest heterogenous impacts of subjective expectations on labor participation: If women with low and medium income hold rational expectations, they will be more likely to work. Our estimates show that for a low-income woman at age 55, the simulated probabilities of working are $45.9 \%$ and $47.5 \%$ under subjective expectations and rational expectations, respectively, a difference of $3.42 \%$. For a medium-income woman of the same age, the difference probability of working is $2.34 \%$. However, women with high incomes would be $6.19 \%$ less likely to work under rational expectations.

The findings from our counterfactual analysis have important policy implications. If a government aims to promote labor participation among women, its policies should distinguish among different income groups. For example, helping low-income women become aware of their actual income evolution potentially might increase their labor force participation, while such an approach might not work for women with high incomes.

\section{Concluding Remarks}

This paper studies dynamic discrete models with agents holding subjective expectations about the state transition, where these beliefs may be different from the objective transition observed in the data. We show that agents' subjective expectations and preferences are nonparametrically identified in both finite and infinite horizon cases. The identification power in finite case comes from variation of CCPs across time, while the identification power in infinite case comes from the information of an additional state variable. We propose to estimate the model using a maximum likelihood estimator, and we present Monte Carlo evidence illustrating that our estimator performs well with mid-sized samples. Applying our method to PSID data, we illustrate that households do not hold rational expectations about their income transitions, and the discrepancies between their subjective expectations and rational expectations may lead to a significant difference in women's labor participation. Our estimates also shed light on how subjective expectations affects agents' dynamic decisions and what policies would be appropriate in order to improve labor participation among women. 
A direction for future research is to relax some important assumptions in this paper, e.g., invariant subjective expectations, and to incorporate learning into the model. While our method is introduced in the context of discrete choice, we might be able to extend it to dynamic models with continuous choices, e.g., life-cycle consumption problems. We are considering these possibilities for future work.

\section{References}

AbBring, J. H. (2010): "Identification of dynamic discrete choice models," Annual Review of Economics, 2(1), 367-394. 1

Abbring, J. H., And Ø. DaLjord (2016): "Identifying the Discount Factor in Dynamic Discrete Choice Models," . 3, 3.1, 4

Aguirregabiria, V., and A. Magesan (2017): "Identification and estimation of dynamic games when players' beliefs are not in equilibrium," Working paper. 1, 2

Aguirregabiria, V., And P. Mira (2002): "Swapping the nested fixed point algorithm: A class of estimators for discrete Markov decision models," Econometrica, 70(4), 15191543. 6.1

(2010): "Dynamic discrete choice structural models: A survey," Journal of Econometrics, 156(1), 38-67. 4

BLEvins, J. R. (2014): "Nonparametric identification of dynamic decision processes with discrete and continuous choices," Quantitative Economics, 5(3), 531-554. 3.1

Chou, C. (2015): "Identification and Linear Estimation of General Dynamic Programming Discrete Choice Models," Mimeo, University of Southern California. 3.1

Cruces, G., R. Perez-Truglia, and M. Tetaz (2013): "Biased perceptions of income distribution and preferences for redistribution: Evidence from a survey experiment," Journal of Public Economics, 98, 100-112. 1. 2

Eckstein, Z., AND K. I. WolPin (1989): "Dynamic labour force participation of married women and endogenous work experience," The Review of Economic Studies, 56(3), 375-390. 7.1 
FAnG, H., AND Y. WANG (2015): "Estimating dynamic discrete choice models with hyperbolic discounting, with an application to mammography decisions," International Economic Review, 56(2), 565-596. 1, 3.1, 4, 4

Heimer, R. Z., K. O. R. Myrseth, and R. S. Schoenle (2017): "Mortality Beliefs and Household Finance Puzzles," . 1, 2, 3.1, 7.2

Hotz, V. J., And R. A. Miller (1993): "Conditional choice probabilities and the estimation of dynamic models," The Review of Economic Studies, 60(3), 497-529. 1. 3.1, 4, 6.1

Hu, Y. (2008): "Identification and Estimation of Nonlinear Models with Misclassification Error Using Instrumental Variables: A General Solution," Journal of Econometrics, 144, 27-61. 1, 5, 5

Hu, Y., AND M. Shum (2012): "Nonparametric identification of dynamic models with unobserved state variables," Journal of Econometrics, 171(1), 32-44. 1, 3.1

Kasahara, H., and K. Shimotsu (2008): "Pseudo-likelihood estimation and bootstrap inference for structural discrete Markov decision models," Journal of Econometrics, 146(1), 92-106. 6.1

Kasahara, H., and K. Shimotsu (2009): "Nonparametric identification of finite mixture models of dynamic discrete choices," Econometrica, 77(1), 135-175. 1, 3.1, 4, 6

Levin, D. A., And Y. Peres (2017): Markov Chains and Mixing Times, vol. 107. American Mathematical Soc. A.2

Magnac, T., and D. Thesmar (2002): "Identifying dynamic discrete decision processes," Econometrica, 70(2), 801-816. 1, 2, 2, 3

Manski, C. (2004): "Measuring expectations," Econometrica, 72(5), 1329-1376. 1. 4

MAnski, C. F. (1993a): "Adolescent econometricians: How do youth infer the returns to schooling?," in Studies of supply and demand in higher education, pp. 43-60. University of Chicago Press. 1 
(1993b): "Dynamic choice in social settings: Learning from the experiences of others," Journal of Econometrics, 58(1-2), 121-136. 2, 2

Norets, A., And X. TANG (2013): "Semiparametric inference in dynamic binary choice models," Review of Economic Studies, 81(3), 1229-1262. 3.1

Rust, J. (1987): "Optimal replacement of GMC bus engines: An empirical model of Harold Zurcher," Econometrica: Journal of the Econometric Society, pp. 999-1033. 2

Rust, J. (1994): "Structural estimation of Markov decision processes," Handbook of econometrics, 4, 3081-3143. 1

VAN DER KlaAuW, W. (2012): "On the use of expectations data in estimating structural dynamic choice models," Journal of Labor Economics, 30(3), 521-554. 1

VAn Der KlaAuw, W., and K. I. Wolpin (2008): "Social security and the retirement and savings behavior of low-income households," Journal of Econometrics, 145(1), 2142. 1

WANG, Y. (2014): "Dynamic implications of subjective expectations: Evidence from adult smokers," American Economic Journal: Applied Economics, 6(1), 1-37. 1, 2, 4

ZAFAR, B. (2011): "How do college students form expectations?," Journal of Labor Economics, 29(2), 301-348. 1

- (2013): "College major choice and the gender gap," Journal of Human Resources, 48(3), 545-595. 1 


\section{Appendix}

\section{A Proofs}

\section{A.1 Proof of Theorem 1}

The proof of Theorem 1 is sketched in the main text. Therefore, we only provide proofs for the main steps used to derive Theorem 1 .

Proof of equation (6). In the dynamic setting we consider, the optimal choice $a_{t}$ in period $t$ is

$$
a_{t}=\arg \max _{a \in \mathcal{A}}\left\{v_{t}(x, a)+\epsilon_{t}(a)\right\}
$$

where $v_{t}\left(x_{t}, a\right)$ is the choice-specific value function. The ex ante value function at $t$ can be expressed as

$$
\begin{aligned}
V_{t}(x) & =\int \sum_{a \in \mathcal{A}} \mathbb{1}\left\{a=a_{t}\right\}\left[v_{t}\left(x_{t}, a\right)+\epsilon_{t}(a)\right] g\left(\epsilon_{t}\right) d \epsilon_{t} \\
& =\log \left\{\sum_{a \in \mathcal{A}} \exp \left[v_{t}(x, a)\right]\right\}
\end{aligned}
$$

where the second equality is obtained under the assumption that $\epsilon_{t}$ is distributed according to a mean zero type-I extreme value distribution. The conditional choice probability is for $i \in \mathcal{A}$

$$
p_{t}(a=i \mid x)=\frac{\exp \left[v_{t}(x, i)\right]}{\sum_{a \in \mathcal{A}} \exp \left[v_{t}(x, a)\right]}
$$

We may further simplify $V_{t}(x)$ with $i=K$ as follows:

$$
\begin{aligned}
V_{t}(x) & =-\log p_{t}(a=K \mid x)+v_{t}(x, a=K) \\
& \equiv-\log p_{t, K}(x)+v_{t, K}(x)
\end{aligned}
$$

Given that the state variable $x$ has support $\mathcal{X}=\{1,2, \ldots, J\}$, we define a row vector of $J-1$ independent subjective expectations as follows:

$$
S_{a}(x)=\left[s\left(x^{\prime}=1 \mid x, a\right), s\left(x^{\prime}=2 \mid x, a\right), \ldots, s\left(x^{\prime}=J-1 \mid x, a\right)\right] .
$$


Notice that $S_{a}(x)$ contains the same information as $s\left(x^{\prime} \mid x, a\right)$. We consider the choicespecific value function

$$
\begin{aligned}
& v_{t}(x, a) \\
= & u(x, a)+\beta \sum_{x^{\prime}=1}^{J} V_{t+1}\left(x^{\prime}\right) s\left(x^{\prime} \mid x, a\right) d x^{\prime} \\
= & u(x, a)+\beta \sum_{x^{\prime}=1}^{J}\left[-\log p_{t+1, K}\left(x^{\prime}\right)+v_{t+1, K}\left(x^{\prime}\right)\right] s\left(x^{\prime} \mid x, a\right) \\
= & u(x, a)+\beta \sum_{x^{\prime}=1}^{J}\left[-\log p_{t+1, K}\left(x^{\prime}\right)+v_{t+1, K}\left(x^{\prime}\right)\right] s\left(x^{\prime} \mid x, a\right) \\
& +\beta\left[-\log p_{t+1, K}(J)+v_{t+1, K}(J)\right]\left[1-\sum_{x^{\prime}=1}^{J-1} s\left(x^{\prime} \mid x, a\right)\right] \\
= & u(x, a)+\beta \sum_{x^{\prime}=1}^{J-1}\left[-\log p_{t+1, K}\left(x^{\prime}\right)+\log p_{t+1, K}(J)\right. \\
& \left.+v_{t+1, K}\left(x^{\prime}\right)-v_{t+1, K}(J)\right] s\left(x^{\prime} \mid x, a\right)+\beta\left[-\log p_{t+1, K}(J)+v_{t+1, K}(J)\right] .
\end{aligned}
$$

We then obtain the choice-specific value function in (6) of the main text.

$$
\begin{aligned}
& v_{t}(x, a) \\
= & u(x, a)+\beta S_{a}(x)\left(-\log \boldsymbol{p}_{t+1, K}+\boldsymbol{v}_{t+1, K}\right)+\beta\left[-\log p_{t+1, K}(J)+v_{t+1, K}(J)\right] .
\end{aligned}
$$

Proof of equation (11). We now focus on the value function corresponding to choice $a=K$ and $\Delta \boldsymbol{v}_{t+1, K}$, where

$$
\begin{aligned}
\Delta \boldsymbol{v}_{t, K} & \equiv \boldsymbol{v}_{t, K}-\boldsymbol{v}_{t-1, K} \\
= & \left(\begin{array}{c}
v_{t, K}(x=1)-v_{t, K}(J) \\
v_{t, K}(x=2)-v_{t, K}(J) \\
\cdots \\
v_{t, K}(x=J-1)-v_{t, K}(J)
\end{array}\right)-\left(\begin{array}{c}
v_{t-1, K}(x=1)-v_{t-1, K}(J) \\
v_{t-1, K}(x=2)-v_{t-1, K}(J) \\
\cdots \\
v_{t-1, K}(x=J-1)-v_{t-1, K}(J)
\end{array}\right) .
\end{aligned}
$$

To further simplify each element of $\Delta \boldsymbol{v}_{t, K}$, we take the difference of (6) for $x_{t}=x$ and $x_{t}=J$ when $a=K$,

$\left[v_{t}(x, K)-v_{t}(J, K)\right]=[u(x, K)-u(J, K)]+\beta\left[\boldsymbol{S}_{K}(x)-\boldsymbol{S}_{K}(J)\right]\left(-\log \boldsymbol{p}_{t+1, K}+\boldsymbol{v}_{t+1, K}\right)$ 
Similarly, for the time period $t-1$ we have

$\left[v_{t-1}(x, K)-v_{t-1}(J, K)\right]=[u(x, K)-u(J, K)]+\beta\left[\boldsymbol{S}_{K}(x)-\boldsymbol{S}_{K}(J)\right]\left(-\log \boldsymbol{p}_{t, K}+\boldsymbol{v}_{t, K}\right)$.

The difference between the two equations above is

$\left[v_{t}(x, K)-v_{t}(J, K)\right]-\left[v_{t-1}(x, K)-v_{t-1}(J, K)\right]=\beta\left[\boldsymbol{S}_{K}(x)-\boldsymbol{S}_{K}(J)\right]\left(-\Delta \log \boldsymbol{p}_{t+1, K}+\Delta \boldsymbol{v}_{t+1, K}\right)$

Rewrite it in matrix form, we obtain equation (11),

$$
\Delta \boldsymbol{v}_{t, K}=\boldsymbol{v}_{t, K}-\boldsymbol{v}_{t-1, K}=\beta \widetilde{\boldsymbol{S}}_{K}\left(-\Delta \log \boldsymbol{p}_{t+1, K}+\Delta \boldsymbol{v}_{t+1, K}\right)
$$

Identification of $\boldsymbol{S}_{i}-\boldsymbol{S}_{K}$ and $\widetilde{\boldsymbol{S}}_{K}$. The DDC model is characterized by the following two sets of equations: The choice probabilities are associated with subjective expectations and value functions through

$$
\Delta \boldsymbol{\xi}_{t, i, K}=\beta\left[\boldsymbol{S}_{i}-\boldsymbol{S}_{K}\right]\left[-\Delta \log \boldsymbol{p}_{t+1, K}+\Delta \boldsymbol{v}_{t+1, K}\right]
$$

and the choice-specific value function evolves according to

$$
\Delta \boldsymbol{v}_{t, K}=\beta \widetilde{\boldsymbol{S}}_{K}\left(-\Delta \log \boldsymbol{p}_{t+1, K}+\Delta \boldsymbol{v}_{t+1, K}\right)
$$

By eliminating the unknown value functions in these two equations, we are able to find the direct relationship between the observed choice probabilities and the subjective expectations. Specifically, the full rank condition in Assumption 4 guarantees the invertibility of $\boldsymbol{S}_{i}-\boldsymbol{S}_{K}$, then from A.3), we have

$$
\left[\boldsymbol{S}_{i}-\boldsymbol{S}_{K}\right]^{-1} \Delta \boldsymbol{\xi}_{t, i, K}=\beta\left(-\Delta \log \boldsymbol{p}_{t+1, K}+\Delta \boldsymbol{v}_{t+1, K}\right) .
$$

Applying the equation above to the period of $t-1$,

$$
\begin{aligned}
{\left[\boldsymbol{S}_{i}-\boldsymbol{S}_{K}\right]^{-1} \Delta \boldsymbol{\xi}_{t-1, i, K} } & =\beta\left(-\Delta \log \boldsymbol{p}_{t, K}+\Delta \boldsymbol{v}_{t, K}\right) \\
& =\beta\left[-\Delta \log \boldsymbol{p}_{t, K}+\beta \widetilde{\boldsymbol{S}}_{K}\left(-\Delta \log \boldsymbol{p}_{t+1, K}+\Delta \boldsymbol{v}_{t+1, K}\right)\right]
\end{aligned}
$$


where the term $\beta\left(-\Delta \log \boldsymbol{p}_{t+1, K}+\Delta \boldsymbol{v}_{t+1, K}\right)$ can be replaced by the left-hand-side of A.5),

$$
\widetilde{\boldsymbol{S}}_{K}\left[\boldsymbol{S}_{i}-\boldsymbol{S}_{K}\right]^{-1} \Delta \boldsymbol{\xi}_{t, i, K}-\beta^{-1}\left[\boldsymbol{S}_{i}-\boldsymbol{S}_{K}\right]^{-1} \Delta \boldsymbol{\xi}_{t-1, i, K}=\Delta \log \boldsymbol{p}_{t, K}
$$

That is

$$
\left[\widetilde{\boldsymbol{S}}_{K}\left[\boldsymbol{S}_{i}-\boldsymbol{S}_{K}\right]^{-1}-\beta^{-1}\left[\boldsymbol{S}_{i}-\boldsymbol{S}_{K}\right]^{-1}\right]\left(\begin{array}{c}
\Delta \boldsymbol{\xi}_{t, i, K} \\
\Delta \boldsymbol{\xi}_{t-1, i, K}
\end{array}\right)=\Delta \log \boldsymbol{p}_{t, K}
$$

This equation implies that the choice probabilities may be directly associated with the subjective expectations. In the equation above, both $\widetilde{\boldsymbol{S}}_{K}$ and $\left[\boldsymbol{S}_{i}-\boldsymbol{S}_{K}\right]^{-1}$ contain $(J-$ $1) \times(J-1)$ unknowns. For a given $t$, the matrix equation contains $J-1$ equations. To identify $\widetilde{\boldsymbol{S}}_{K}$ and $\boldsymbol{S}_{i}-\boldsymbol{S}_{K}$, we augment A.7) by varying time to introduce more equations. Suppose that we observe data for $2 J-2$ periods. Define a $(2 J-2) \times(2 J-2)$ matrix

$$
\Delta \boldsymbol{\xi}_{i, K} \equiv\left[\begin{array}{cccc}
\Delta \boldsymbol{\xi}_{t_{1}, i, K} & \Delta \boldsymbol{\xi}_{t_{2}, i, K} & \ldots & \Delta \boldsymbol{\xi}_{t_{2 J-2}, i, K} \\
\Delta \boldsymbol{\xi}_{t_{1}-1, i, K} & \Delta \boldsymbol{\xi}_{t_{2}-1, i, K} & \ldots & \Delta \boldsymbol{\xi}_{t_{2 J-3}, i, K}
\end{array}\right]
$$

and a $(J-1) \times(2 J-2)$ matrix

$$
\Delta \log \boldsymbol{p}_{K} \equiv\left[\Delta \log \boldsymbol{p}_{t_{1}, K}, \Delta \log \boldsymbol{p}_{t_{2}, K}, \ldots, \Delta \log \boldsymbol{p}_{t_{2 J-2}, K}\right],
$$

where both $\Delta \boldsymbol{\xi}_{i, K}$ and $\Delta \log \boldsymbol{p}_{K}$ are observed directly from data. Now $(J-1) \times(2 J-2)$ equations are available, and we organize them into a matrix equation,

$$
\left[\widetilde{\boldsymbol{S}}_{K}\left[\boldsymbol{S}_{i}-\boldsymbol{S}_{K}\right]^{-1}, \quad-\beta^{-1}\left[\boldsymbol{S}_{i}-\boldsymbol{S}_{K}\right]^{-1}\right] \Delta \boldsymbol{\xi}_{i, K}=\Delta \log \boldsymbol{p}_{K},
$$

where $\left[\widetilde{\boldsymbol{S}}_{K}\left[\boldsymbol{S}_{i}-\boldsymbol{S}_{K}\right]^{-1}, \quad-\beta^{-1}\left[\boldsymbol{S}_{i}-\boldsymbol{S}_{K}\right]^{-1}\right]$ is a matrix of dimension of $(J-1) \times(2 J-2)$.

Under Assumption 5A. $\left[\boldsymbol{S}_{i}-\boldsymbol{S}_{K}\right]$ and $\widetilde{\boldsymbol{S}}_{K}$ are solved with a closed-form expression with a known $\beta$ as follows:

$$
\left[\widetilde{\boldsymbol{S}}_{K}\left[\boldsymbol{S}_{i}-\boldsymbol{S}_{K}\right]^{-1}, \quad-\beta^{-1}\left[\boldsymbol{S}_{i}-\boldsymbol{S}_{K}\right]^{-1}\right]=\Delta \log \boldsymbol{p}_{K}\left[\Delta \boldsymbol{\xi}_{i, K}\right]^{-1},
$$

Given the definition of $\widetilde{\boldsymbol{S}}_{K}$, we have identified $\boldsymbol{S}_{K}(x)-\boldsymbol{S}_{K}(J)$ for $x \in\{1,2, \ldots, J-1\}$. Assumption 6A normalizes $\boldsymbol{S}_{K}(J)$ to a known distribution, and therefore, we fully recover $\boldsymbol{S}_{K}(x)$ for $x \in\{1,2, \ldots, J\}$, i.e., $\boldsymbol{S}_{K}$. Therefore, all the subjective probabilities $\boldsymbol{S}_{i}$ 
are identified from $\boldsymbol{S}_{i}-\boldsymbol{S}_{K}$ with a closed-form. QED.

\section{A.2 Proof of Corollary 1}

Proof of equation (19) Considering $u(x, K)=0$, we can derive the following expression of agents' choice-specific value function for choice $K$,

$$
\begin{aligned}
v_{t}(x, K) & =\beta \sum_{x^{\prime}=1}^{J}\left[-\log p_{t+1, K}\left(x^{\prime}\right)+v_{t+1, K}\left(x^{\prime}\right)\right] s\left(x^{\prime} \mid x, K\right) \\
& =\beta S_{K}^{\dagger}(x)\left[-\log \boldsymbol{p}_{t+1, K}^{\dagger}+\boldsymbol{v}_{t+1, K}^{\dagger}\right] \\
& =\beta S_{K}^{\dagger}(x)\left(-\log \boldsymbol{p}_{t+1, K}^{\dagger}\right)+\left[\beta S_{K}^{\dagger}(x)\right]^{2}\left[-\log \boldsymbol{p}_{t+2, K}^{\dagger}+\boldsymbol{v}_{t+2, K}^{\dagger}\right] \\
& \vdots \\
& =\sum_{\tau=t+1}^{T}\left[-\beta S_{K}^{\dagger}(x)\right]^{\tau-t}\left(\log \boldsymbol{p}_{\tau, K}^{\dagger}\right)+\left[\beta S_{K}^{\dagger}(x)\right]^{T-t} \boldsymbol{v}_{T, K}^{\dagger}, \quad t \neq T
\end{aligned}
$$

Similarly, for all other choices $a \neq K$,

$$
\begin{aligned}
v_{t}(x, a) & =u(x, a)+\beta S_{a}^{\dagger}(x)\left[-\log \boldsymbol{p}_{t+1, K}^{\dagger}+\boldsymbol{v}_{t+1, K}^{\dagger}\right] \\
& =\log p_{t, a}-\log p_{t, K}+v_{t}(x, K) .
\end{aligned}
$$

Applying both the iterated expression in A.12 and the Hotz-Miller relationship, we obtain

$$
\begin{aligned}
& u(x, a)-\beta\left[S_{a}^{\dagger}(x)-S_{K}^{\dagger}(x)\right] \sum_{\tau=t+2}^{T}\left[-\beta S_{K}^{\dagger}(x)\right]^{\tau-t-1}\left(\log \boldsymbol{p}_{\tau, K}^{\dagger}\right) \\
= & \log p_{t, a}(x)-\log p_{t, K}(x)+\beta\left[S_{a}^{\dagger}(x)-S_{K}^{\dagger}(x)\right] \log \boldsymbol{p}_{t+1, K}^{\dagger}+\left[\beta S_{K}^{\dagger}(x)\right]^{T-t-1} \boldsymbol{v}_{T, K}^{\dagger} .
\end{aligned}
$$

The equation above builds up a link between an agent's preference and the value at the last period, and it holds for $t=1,2, \cdots T-2, x=1,2, \cdots, J$. Using the matrix notation 
below,

$$
\boldsymbol{u}_{a} \equiv\left(\begin{array}{c}
u(x=1, a) \\
u(x=2, a) \\
\vdots \\
u(x=J, a)
\end{array}\right) ; \boldsymbol{S}_{a}^{\dagger}=\left(\begin{array}{c}
S_{a}^{\dagger}(x=1) \\
S_{a}^{\dagger}(x=2) \\
\vdots \\
S_{a}^{\dagger}(x=J)
\end{array}\right), a \in \mathcal{A}
$$

we obtain a matrix form of A.13

$$
\begin{aligned}
& \boldsymbol{u}_{a}-\beta\left(\boldsymbol{S}_{a}^{\dagger}-\boldsymbol{S}_{K}^{\dagger}\right) \sum_{\tau=t+2}^{T}\left(-\beta \boldsymbol{S}_{K}^{\dagger}\right)^{\tau-t-1}\left(\log \boldsymbol{p}_{\tau, K}^{\dagger}\right) \\
= & \log \boldsymbol{p}_{t, a}^{\dagger}-\log \boldsymbol{p}_{t, K}^{\dagger}+\beta\left(\boldsymbol{S}_{a}^{\dagger}-\boldsymbol{S}_{K}^{\dagger}\right) \log \boldsymbol{p}_{t+1, K}^{\dagger}+\left(\beta \boldsymbol{S}_{K}^{\dagger}\right)^{T-t-1} \boldsymbol{v}_{T, K}^{\dagger}
\end{aligned}
$$

Consider another time period $t+t^{\prime} \in\{2, \cdots, T-2\}$ with $t^{\prime} \in\{1,2, \cdots, T-3\}$, the equation above is

$$
\begin{aligned}
& \boldsymbol{u}_{a}-\beta\left(\boldsymbol{S}_{a}^{\dagger}-\boldsymbol{S}_{K}^{\dagger}\right) \sum_{\tau=t+t^{\prime}+2}^{T}\left(-\beta \boldsymbol{S}_{K}^{\dagger}\right)^{\tau-t-t^{\prime}-1}\left(\log \boldsymbol{p}_{\tau, K}^{\dagger}\right) \\
= & \log \boldsymbol{p}_{t+t^{\prime}, a}^{\dagger}-\log \boldsymbol{p}_{t+t^{\prime}, K}^{\dagger}+\beta\left(\boldsymbol{S}_{a}^{\dagger}-\boldsymbol{S}_{K}^{\dagger}\right) \log \boldsymbol{p}_{t+t^{\prime}+1, K}^{\dagger}+\left(\beta \boldsymbol{S}_{K}^{\dagger}\right)^{T-t-t^{\prime}-1} \boldsymbol{v}_{T, K}^{\dagger} .
\end{aligned}
$$

Eliminating the unknown value $\boldsymbol{v}_{T, K}^{\dagger}$ from the two equations above, we obtain

$$
\begin{aligned}
& {\left[I-\left(\beta \boldsymbol{S}_{K}^{\dagger}\right)^{t^{\prime}}\right] \boldsymbol{u}_{a} } \\
= & \beta\left(\boldsymbol{S}_{a}^{\dagger}-\boldsymbol{S}_{K}^{\dagger}\right)\left[\sum_{\tau=t+2}^{T}\left(-\beta \boldsymbol{S}_{K}^{\dagger}\right)^{\tau-t-1}\left(\log \boldsymbol{p}_{\tau, K}^{\dagger}\right)-\sum_{\tau=t+t^{\prime}+2}^{T}\left(-\beta \boldsymbol{S}_{K}^{\dagger}\right)^{\tau-t-t^{\prime}-1}\left(\log \boldsymbol{p}_{\tau, K}^{\dagger}\right)\right] \\
& +\log \boldsymbol{p}_{t, a}^{\dagger}-\log \boldsymbol{p}_{t, K}^{\dagger}+\beta\left(\boldsymbol{S}_{a}^{\dagger}-\boldsymbol{S}_{K}^{\dagger}\right) \log \boldsymbol{p}_{t+1, K}^{\dagger} \\
& -\left(\beta \boldsymbol{S}_{k}^{+}\right)^{t^{\prime}}\left[\log \boldsymbol{p}_{t+t^{\prime}, a}^{\dagger}-\log \boldsymbol{p}_{t+t^{\prime}, K}^{\dagger}+\beta\left(\boldsymbol{S}_{a}^{\dagger}-\boldsymbol{S}_{K}^{\dagger}\right) \log \boldsymbol{p}_{t+t^{\prime}+1, K}^{\dagger}\right]
\end{aligned}
$$

It is clear from A.16 that $\boldsymbol{u}_{a}$ is identified if $I-\left(\beta \boldsymbol{S}_{K}^{\dagger}\right)^{t^{\prime}}$ is invertible since all the terms on the right-hand-side of this equation are known. Recall that $\boldsymbol{S}_{K}^{\dagger}$ is a Markov transition matrix with each row summing up to one. Therefore all the eigenvalues of $\boldsymbol{S}_{K}^{\dagger}$ have absolute values being less than or equal to 1 , and 1 is an eigenvalue of $\boldsymbol{S}_{K}^{\dagger}$ (see e.g., Levin and Peres (2017), p.160). Let us denote the $J$ eigenvalues of $\boldsymbol{S}_{K}^{\dagger}$ as $\lambda_{1}, \cdots, \lambda_{J}$ such that

$$
0 \leq\left|\lambda_{1}\right| \leq\left|\lambda_{2}\right| \leq \cdots\left|\lambda_{J}\right|=1
$$


For any discount factor $\beta \in(0,1)$ and $t^{\prime} \in\{1,2, \cdots, T-3\}$, the eigenvalues of $\left(\beta \boldsymbol{S}_{K}^{\dagger}\right)^{t^{\prime}}$ are $\left(\beta \lambda_{1}\right)^{t^{\prime}}, \cdots,\left(\beta \lambda_{J}\right)^{t^{\prime}}$. From A.17), we have

$$
0 \leq\left|\left(\beta \lambda_{1}\right)^{t^{\prime}}\right| \leq \cdots\left|\left(\beta \lambda_{J}\right)^{t^{\prime}}\right|<1
$$

The eigenvalues of matrix $I-\left(\beta \boldsymbol{S}_{K}^{\dagger}\right)^{t^{\prime}}$ are $1-\left(\beta \lambda_{1}\right)^{t^{\prime}}, \cdots, 1-\left(\beta \lambda_{J}\right)^{t^{\prime}}$. It is clear from A.18 that all the eigenvalues of $I-\left(\beta \boldsymbol{S}_{K}^{\dagger}\right)^{t^{\prime}}$ are nonzero. Therefore, $I-\left(\beta \boldsymbol{S}_{K}^{\dagger}\right)^{t^{\prime}}$ is an invertible matrix, and $\boldsymbol{u}_{a}$ can be identified by multiplying $\left[I-\left(\beta \boldsymbol{S}_{K}^{\dagger}\right)^{t^{\prime}}\right]^{-1}$ from left to the right-hand-side of A.16). This proof holds for all $a \in \mathcal{A}$ and $a \neq K$. QED.

\section{A.3 Proof of Theorem 4}

The first-order Markov process $\left\{a_{t}, x_{t}, \tau\right\}$ indiciates

$$
\operatorname{Pr}\left(a_{t+}, x_{t+1}, a_{t}, x_{t}, a_{t-}\right)=\sum_{\tau=1}^{H} \operatorname{Pr}\left(a_{t+} \mid x_{t+1}, \tau\right) \operatorname{Pr}\left(x_{t+1}, a_{t} \mid x_{t}, \tau\right) \operatorname{Pr}\left(\tau, x_{t}, a_{t-}\right),
$$

with $a_{t+}=h\left(a_{t+l}, \ldots, a_{t+1}\right)$ and $a_{t-}=h\left(a_{t-1}, \ldots, a_{t-l}\right)$.

Note that we have reduced the support of $a_{t+l}, \ldots, a_{t+1}$ to that of $\tau$ by the mapping $h(\cdot)$. We define the following matrices for given $x_{t}, x_{t+1}$ and $a_{t}=k$,

$$
\begin{aligned}
M_{a_{t+}, x_{t+1}, k, x_{t}, a_{t-}} & =\left[\operatorname{Pr}\left(a_{t+}=i, x_{t+1}, k, x_{t}, a_{t-}=j\right)\right]_{i, j} \\
M_{a_{t+}, x_{t+1}, \tau} & =\left[\operatorname{Pr}\left(a_{t+}=i \mid x_{t+1}, \tau=j\right)\right]_{i, j} \\
M_{\tau, x_{t}, a_{t-}} & =\left[\operatorname{Pr}\left(\tau=i, x_{t}, a_{t-}=j\right)\right]_{i, j} \\
D_{x_{t+1}, k \mid x_{t}, \tau} & =\operatorname{diag}\left\{\operatorname{Pr}\left(x_{t+1}, k \mid x_{t}, \tau=1\right), \ldots, \operatorname{Pr}\left(x_{t+1}, k \mid x_{t}, \tau=L\right)\right\} \\
D_{k \mid x_{t+1}, x_{t}, \tau} & =\operatorname{diag}\left\{\operatorname{Pr}\left(k \mid x_{t+1}, x_{t}, \tau=1\right), \ldots, \operatorname{Pr}\left(k \mid x_{t+1}, x_{t}, \tau=L\right)\right\} .
\end{aligned}
$$

Equation A.19 is equivalent to

$$
M_{a_{t+}, x_{t+1}, k, x_{t}, a_{t-}}=M_{a_{t+} \mid x_{t+1}, \tau} D_{x_{t+1}, k \mid x_{t}, \tau} M_{\tau, x_{t}, a_{t-}} .
$$


Similarly, we have

$$
M_{a_{t+}, x_{t+1}, x_{t}, a_{t-}}=M_{a_{t+} \mid x_{t+1}, \tau} D_{x_{t+1} \mid x_{t}} M_{\tau, x_{t}, a_{t-}},
$$

where the matrices are defined analogously to those in A.20 based on the following equality

$$
\begin{aligned}
& \sum_{a_{t}=1}^{K} \operatorname{Pr}\left(a_{t+}, x_{t+1}, a_{t}, x_{t}, a_{t-}\right) \\
= & \sum_{\tau=1}^{L} \operatorname{Pr}\left(a_{t+} \mid x_{t+1}, \tau\right)\left[\sum_{a_{t}=1}^{K} \operatorname{Pr}\left(x_{t+1}, a_{t} \mid x_{t}, \tau\right)\right] \operatorname{Pr}\left(\tau, x_{t}, a_{t-}\right) \\
= & \sum_{\tau=1}^{L} \operatorname{Pr}\left(a_{t+} \mid x_{t+1}, \tau\right) \operatorname{Pr}\left(x_{t+1} \mid x_{t}, \tau\right) \operatorname{Pr}\left(\tau, x_{t}, a_{t-}\right) .
\end{aligned}
$$

Assumption 12 implies that matrices $M_{a_{t+} \mid x_{t+1}, \tau}$ and $M_{\tau, x_{t}, a_{t-}}$ are both invertible. We may then consider

$$
\begin{aligned}
M_{a_{t+}, x_{t+1}, k, x_{t}, a_{t-}} M_{a_{t+}, x_{t+1}, x_{t}, a_{t-}}^{-1} & =M_{a_{t+} \mid x_{t+1}, \tau} D_{x_{t+1}, k \mid x_{t}, \tau} D_{x_{t+1} \mid x_{t}}^{-1} M_{a_{t+}, x_{t+1}, \tau}^{-1} \\
& \equiv M_{a_{t+} \mid x_{t+1}, \tau} D_{k \mid x_{t+1}, x_{t}, \tau} M_{a_{t+}, x_{t+1}, \tau}^{-1}
\end{aligned}
$$

This equation above shows an eigenvalue-eigenvector decomposition of an observed matrix on the left-hand side. Assumptions 13 and 14 guarantee that this decomposition is unique. Therefore, the eigenvector matrix $M_{a_{t+} \mid x_{t+1}, \tau}$, i.e., $\operatorname{Pr}\left(a_{t+} \mid x_{t+1}, \tau\right)$ is identified. We can recover the matrix $M_{\tau, x_{t}, a_{t-}}$ from (A.22). The distribution $f\left(x_{t+1}, a_{t} \mid x_{t}, \tau\right)$, and therefore $\operatorname{Pr}\left(a_{t} \mid x_{t}, \tau\right)=p_{t}\left(a_{t} \mid x_{t}, \tau\right)$ by integrate out $x_{t+1}$, can then identified from equation A.19 due to the invertibility of matrix $M_{a_{t+}, x_{t+1}, \tau}$. QED. 


\section{B Tables}

Table 1: Finite Horizon: normalization of $\boldsymbol{S}_{1}\left(x_{3}\right)$ (DGP: rational expectations)

\begin{tabular}{|c|c|c|c|c|c|c|c|c|c|}
\hline & & \multicolumn{4}{|c|}{ Estimates with subjective beliefs } & \multicolumn{4}{|c|}{ Estimates with rational expectation } \\
\hline & True & $\mathrm{N}=300$ & $\mathrm{~N}=600$ & $\mathrm{~N}=1000$ & $\mathrm{~N}=2500$ & $\mathrm{~N}=300$ & $\mathrm{~N}=600$ & $\mathrm{~N}=1000$ & $\mathrm{~N}=2500$ \\
\hline \multirow[t]{2}{*}{$u_{1}$} & -2 & -2.04 & -2.01 & -2.01 & -2.01 & -2.01 & -2.00 & -2.00 & -2.01 \\
\hline & & $(0.23)$ & $(0.16)$ & $(0.13)$ & $(0.08)$ & $(0.16)$ & $(0.12)$ & $(0.09)$ & $(0.06)$ \\
\hline \multirow{2}{*}{$u_{2}$} & 0.4 & 0.38 & 0.39 & 0.40 & 0.41 & 0.40 & 0.40 & 0.41 & 0.40 \\
\hline & & $(0.24)$ & $(0.16)$ & $(0.12)$ & $(0.08)$ & $(0.18)$ & $(0.13)$ & $(0.11)$ & $(0.06)$ \\
\hline \multirow[t]{2}{*}{$u_{3}$} & 2.1 & 2.16 & 2.16 & 2.13 & 2.12 & 2.10 & 2.10 & 2.10 & 2.10 \\
\hline & & $(0.36)$ & $(0.25)$ & $(0.18)$ & $(0.11)$ & $(0.25)$ & $(0.18)$ & $(0.12)$ & $(0.08)$ \\
\hline \multirow[t]{2}{*}{$\mathrm{S} 0(1,1)$} & 0.9 & 0.85 & 0.85 & 0.85 & 0.86 & & & & \\
\hline & & $(0.12)$ & $(0.08)$ & $(0.08)$ & $(0.04)$ & & & & \\
\hline \multirow[t]{2}{*}{$\mathrm{S} 0(2,1)$} & 0.07 & 0.22 & 0.24 & 0.21 & 0.22 & & & & \\
\hline & & $(0.21)$ & $(0.21)$ & $(0.20)$ & $(0.18)$ & & & & \\
\hline \multirow[t]{2}{*}{$\mathrm{S} 0(3,1)$} & 0.03 & 0.03 & 0.03 & 0.03 & 0.03 & & & & \\
\hline & & $(0.04)$ & $(0.04)$ & $(0.03)$ & $(0.03)$ & & & & \\
\hline \multirow[t]{2}{*}{$\mathrm{S} 0(1,2)$} & 0.03 & 0.10 & 0.11 & 0.10 & 0.08 & & & & \\
\hline & & $(0.14)$ & $(0.11)$ & $(0.10)$ & $(0.07)$ & & & & \\
\hline \multirow[t]{2}{*}{$\mathrm{S} 0(2,2)$} & 0.87 & 0.56 & 0.52 & 0.57 & 0.57 & & & & \\
\hline & & $(0.43)$ & $(0.41)$ & $(0.39)$ & $(0.37)$ & & & & \\
\hline \multirow[t]{2}{*}{$\mathrm{S} 0(3,2)$} & 0.06 & 0.07 & 0.06 & 0.06 & 0.06 & & & & \\
\hline & & $(0.11)$ & $(0.08)$ & $(0.07)$ & $(0.05)$ & & & & \\
\hline \multirow[t]{2}{*}{$\mathrm{S} 1(1,1)$} & 0.2 & 0.25 & 0.23 & 0.24 & 0.22 & & & & \\
\hline & & $(0.22)$ & $(0.22)$ & $(0.21)$ & $(0.18)$ & & & & \\
\hline \multirow[t]{2}{*}{$\mathrm{S} 1(2,1)$} & 0.5 & 0.39 & 0.38 & 0.40 & 0.43 & & & & \\
\hline & & $(0.21)$ & $(0.19)$ & $(0.16)$ & $(0.14)$ & & & & \\
\hline \multirow[t]{2}{*}{$\mathrm{S} 1(1,2)$} & 0.6 & 0.50 & 0.54 & 0.52 & 0.57 & & & & \\
\hline & & $(0.45)$ & $(0.43)$ & $(0.41)$ & $(0.36)$ & & & & \\
\hline \multirow[t]{2}{*}{$\mathrm{S} 1(2,2)$} & 0.2 & 0.34 & 0.35 & 0.33 & 0.29 & & & & \\
\hline & & $(0.35)$ & $(0.33)$ & $(0.29)$ & $(0.24)$ & & & & \\
\hline
\end{tabular}


Table 2: Finite Horizon: normalization of $\boldsymbol{S}_{1}\left(x_{3}\right)$ (DGP: subjective beliefs)

\begin{tabular}{|c|c|c|c|c|c|c|c|c|c|}
\hline & & \multicolumn{4}{|c|}{ Estimates with subjective beliefs } & \multicolumn{4}{|c|}{ Estimates with rational expectation } \\
\hline & True & $\mathrm{N}=300$ & $\mathrm{~N}=600$ & $\mathrm{~N}=1000$ & $\mathrm{~N}=2500$ & $\mathrm{~N}=300$ & $\mathrm{~N}=600$ & $\mathrm{~N}=1000$ & $\mathrm{~N}=2500$ \\
\hline \multirow[t]{2}{*}{$u_{1}$} & -2 & -2.02 & -2.00 & -2.00 & -2.00 & -2.55 & -2.54 & -2.54 & -2.53 \\
\hline & & $(0.21)$ & $(0.15)$ & $(0.12)$ & $(0.07)$ & $(0.19)$ & $(0.15)$ & $(0.11)$ & $(0.07)$ \\
\hline \multirow[t]{2}{*}{$u_{2}$} & 0.4 & 0.43 & 0.40 & 0.40 & 0.40 & 0.25 & 0.24 & 0.25 & 0.24 \\
\hline & & $(0.27)$ & $(0.19)$ & $(0.13)$ & $(0.09)$ & $(0.20)$ & $(0.14)$ & $(0.11)$ & $(0.06)$ \\
\hline \multirow[t]{2}{*}{$u_{3}$} & 2.1 & 2.22 & 2.20 & 2.15 & 2.13 & 2.54 & 2.56 & 2.56 & 2.56 \\
\hline & & $(0.59)$ & $(0.37)$ & $(0.26)$ & $(0.16)$ & $(0.30)$ & $(0.21)$ & $(0.15)$ & $(0.10)$ \\
\hline \multirow[t]{2}{*}{$\mathrm{S} 0(1,1)$} & 0.7 & 0.55 & 0.57 & 0.58 & 0.60 & & & & \\
\hline & & $(0.23)$ & $(0.21)$ & $(0.18)$ & $(0.15)$ & & & & \\
\hline \multirow[t]{2}{*}{$\mathrm{S} 0(2,1)$} & 0.2 & 0.29 & 0.29 & 0.30 & 0.33 & & & & \\
\hline & & $(0.20)$ & $(0.19)$ & $(0.17)$ & $(0.15)$ & & & & \\
\hline \multirow[t]{2}{*}{$\mathrm{S} 0(3,1)$} & 0.1 & 0.11 & 0.09 & 0.10 & 0.10 & & & & \\
\hline & & $(0.15)$ & $(0.11)$ & $(0.10)$ & $(0.09)$ & & & & \\
\hline \multirow[t]{2}{*}{$\mathrm{S} 0(1,2)$} & 0.1 & 0.28 & 0.26 & 0.25 & 0.23 & & & & \\
\hline & & $(0.29)$ & $(0.28)$ & $(0.25)$ & $(0.23)$ & & & & \\
\hline \multirow[t]{2}{*}{$\mathrm{S} 0(2,2)$} & 0.6 & 0.43 & 0.42 & 0.39 & 0.35 & & & & \\
\hline & & $(0.41)$ & $(0.38)$ & $(0.35)$ & $(0.31)$ & & & & \\
\hline \multirow[t]{2}{*}{$\mathrm{S} 0(3,2)$} & 0.19 & 0.20 & 0.19 & 0.17 & 0.18 & & & & \\
\hline & & $(0.26)$ & $(0.19)$ & $(0.17)$ & $(0.14)$ & & & & \\
\hline \multirow[t]{2}{*}{$\mathrm{S} 1(1,1)$} & 0.2 & 0.26 & 0.26 & 0.27 & 0.26 & & & & \\
\hline & & $(0.22)$ & $(0.21)$ & $(0.21)$ & $(0.20)$ & & & & \\
\hline \multirow[t]{2}{*}{$\mathrm{S} 1(2,1)$} & 0.5 & 0.38 & 0.36 & 0.36 & 0.36 & & & & \\
\hline & & $(0.26)$ & $(0.22)$ & $(0.20)$ & $(0.18)$ & & & & \\
\hline \multirow[t]{2}{*}{$\mathrm{S} 1(1,2)$} & 0.6 & 0.48 & 0.48 & 0.45 & 0.47 & & & & \\
\hline & & $(0.43)$ & $(0.42)$ & $(0.41)$ & $(0.39)$ & & & & \\
\hline \multirow[t]{2}{*}{$\mathrm{S} 1(2,2)$} & 0.2 & 0.38 & 0.39 & 0.40 & 0.38 & & & & \\
\hline & & $(0.33)$ & $(0.33)$ & $(0.32)$ & $(0.29)$ & & & & \\
\hline
\end{tabular}


Table 3: Finite Horizon: normalization of $\boldsymbol{S}_{K}$ (DGP: rational expectations)

\begin{tabular}{|c|c|c|c|c|c|c|c|c|c|}
\hline & & Estim & ites with & subjective & beliefs & Estima & es with & tional ex & ectation \\
\hline & True & $\mathrm{N}=300$ & $\mathrm{~N}=600$ & $\mathrm{~N}=1000$ & $\mathrm{~N}=2500$ & $\mathrm{~N}=300$ & $\mathrm{~N}=600$ & $\mathrm{~N}=1000$ & $\mathrm{~N}=2500$ \\
\hline$u_{1}$ & -2 & -2.01 & -2.00 & -2.01 & -2.01 & -2.01 & -2.00 & -2.00 & -2.01 \\
\hline & & $(0.22)$ & $(0.15)$ & $(0.12)$ & $(0.07)$ & $(0.16)$ & $(0.12)$ & $(0.09)$ & $(0.06)$ \\
\hline$u_{2}$ & 0.4 & 0.42 & 0.42 & 0.42 & 0.41 & 0.40 & 0.40 & 0.41 & 0.40 \\
\hline & & $(0.18)$ & $(0.14)$ & $(0.12)$ & $(0.07)$ & $(0.18)$ & $(0.13)$ & $(0.11)$ & $(0.06)$ \\
\hline$u_{3}$ & 2.1 & 2.16 & 2.13 & 2.13 & 2.12 & 2.10 & 2.10 & 2.10 & 2.10 \\
\hline & & $(0.34)$ & $(0.24)$ & $(0.18)$ & $(0.11)$ & $(0.25)$ & $(0.18)$ & $(0.12)$ & $(0.08)$ \\
\hline $\mathrm{S} 0(1,1)$ & 0.9 & 0.84 & 0.85 & 0.86 & 0.87 & & & & \\
\hline & & $(0.13)$ & $(0.08)$ & $(0.08)$ & $(0.05)$ & & & & \\
\hline $\mathrm{S} 0(2,1)$ & 0.07 & 0.21 & 0.18 & 0.15 & 0.11 & & & & \\
\hline & & $(0.21)$ & $(0.20)$ & $(0.18)$ & $(0.12)$ & & & & \\
\hline $\mathrm{S} 0(3,1)$ & 0.03 & 0.03 & 0.03 & 0.03 & 0.04 & & & & \\
\hline & & $(0.05)$ & $(0.05)$ & $(0.04)$ & $(0.04)$ & & & & \\
\hline $\mathrm{S} 0(1,2)$ & 0.03 & 0.12 & 0.10 & 0.10 & 0.09 & & & & \\
\hline & & $(0.14)$ & $(0.11)$ & $(0.10)$ & $(0.08)$ & & & & \\
\hline $\mathrm{S} 0(2,2)$ & 0.87 & 0.59 & 0.65 & 0.70 & 0.78 & & & & \\
\hline & & $(0.43)$ & $(0.39)$ & $(0.36)$ & $(0.24)$ & & & & \\
\hline $\mathrm{S} 0(3,2)$ & 0.06 & 0.07 & 0.06 & 0.06 & 0.05 & & & & \\
\hline & & $(0.12)$ & $(0.08)$ & $(0.08)$ & $(0.06)$ & & & & \\
\hline
\end{tabular}

Table 4: Finite Horizon: normalization of $\boldsymbol{S}_{K}$ (DGP: subjective beliefs)

\begin{tabular}{|c|c|c|c|c|c|c|c|c|c|}
\hline & & \multicolumn{4}{|c|}{ Estimates with subjective beliefs } & \multicolumn{4}{|c|}{ Estimates with rational expectation } \\
\hline & True & $\mathrm{N}=300$ & $\mathrm{~N}=600$ & $\mathrm{~N}=1000$ & $\mathrm{~N}=2500$ & $\mathrm{~N}=300$ & $\mathrm{~N}=600$ & $\mathrm{~N}=1000$ & $\mathrm{~N}=2500$ \\
\hline \multirow{2}{*}{$u_{1}$} & -2 & -2.00 & -1.99 & -2.00 & -2.00 & -2.55 & -2.54 & -2.54 & -2.53 \\
\hline & & $(0.22)$ & $(0.16)$ & $(0.13)$ & $(0.08)$ & (0.19) & $(0.15)$ & $(0.11)$ & $(0.07)$ \\
\hline \multirow[t]{2}{*}{$u_{2}$} & 0.4 & 0.39 & 0.40 & 0.40 & 0.40 & 0.25 & 0.24 & 0.25 & 0.24 \\
\hline & & $(0.20)$ & $(0.14)$ & $(0.12)$ & $(0.08)$ & $(0.20)$ & $(0.14)$ & $(0.11)$ & $(0.06)$ \\
\hline \multirow[t]{2}{*}{$u_{3}$} & 2.1 & 2.20 & 2.14 & 2.12 & 2.11 & 2.54 & 2.56 & 2.56 & 2.56 \\
\hline & & $(0.40)$ & $(0.27)$ & $(0.19)$ & $(0.12)$ & $(0.30)$ & $(0.21)$ & $(0.15)$ & $(0.10)$ \\
\hline \multirow[t]{2}{*}{$\mathrm{S} 0(1,1)$} & 0.7 & 0.53 & 0.55 & 0.56 & 0.59 & & & & \\
\hline & & $(0.25)$ & $(0.22)$ & $(0.22)$ & $(0.18)$ & & & & \\
\hline \multirow[t]{2}{*}{$\mathrm{S} 0(2,1)$} & 0.2 & 0.24 & 0.24 & 0.22 & 0.22 & & & & \\
\hline & & $(0.20)$ & $(0.18)$ & $(0.15)$ & $(0.11)$ & & & & \\
\hline \multirow[t]{2}{*}{$\mathrm{S} 0(3,1)$} & 0.1 & 0.10 & 0.10 & 0.11 & 0.11 & & & & \\
\hline & & $(0.12)$ & $(0.11)$ & $(0.10)$ & $(0.09)$ & & & & \\
\hline \multirow[t]{2}{*}{$\mathrm{S} 0(1,2)$} & 0.1 & 0.34 & 0.32 & 0.31 & 0.27 & & & & \\
\hline & & $(0.33)$ & $(0.31)$ & $(0.30)$ & $(0.26)$ & & & & \\
\hline \multirow[t]{2}{*}{$\mathrm{S} 0(2,2)$} & 0.6 & 0.52 & 0.52 & 0.55 & 0.56 & & & & \\
\hline & & $(0.40)$ & $(0.35)$ & $(0.31)$ & $(0.22)$ & & & & \\
\hline \multirow[t]{2}{*}{$\mathrm{S} 0(3,2)$} & 0.19 & 0.18 & 0.19 & 0.17 & 0.18 & & & & \\
\hline & & $(0.22)$ & $(0.18)$ & $(0.16)$ & $(0.14)$ & & & & \\
\hline
\end{tabular}


Table 5: Infinite Horizon: normalization of $\boldsymbol{S}_{1}^{\boldsymbol{x}}$ (DGP: rational expectations)

\begin{tabular}{cccccc|cccc}
\hline \hline & \multicolumn{1}{c|}{ Estimates with Subjective beliefs } & \multicolumn{3}{c}{ Estimates with Rational Expectation } \\
\hline \multirow{4}{*}{$\left.u_{1}(x)\right)$} & TRUE & $\mathrm{N}=300$ & $\mathrm{~N}=600$ & $\mathrm{~N}=1000$ & $\mathrm{~N}=2500$ & $\mathrm{~N}=300$ & $\mathrm{~N}=600$ & $\mathrm{~N}=1000$ & $\mathrm{~N}=2500$ \\
& 0.1 & 0.08 & 0.09 & 0.09 & 0.09 & 0.10 & 0.11 & 0.10 & 0.10 \\
$u_{1}(x 2)$ & 0.2 & $(0.16)$ & $(0.11)$ & $(0.08)$ & $(0.06)$ & $(0.17)$ & $(0.12)$ & $(0.09)$ & $(0.06)$ \\
& & 0.21 & 0.21 & 0.21 & 0.20 & 0.20 & 0.20 & 0.20 & 0.20 \\
$u_{2}(x 1)$ & 0.2 & $0.18)$ & $(0.12)$ & $(0.10)$ & $(0.07)$ & $(0.18)$ & $(0.13)$ & $(0.10)$ & $(0.06)$ \\
& & $(0.09)$ & $(0.16$ & 0.17 & 0.18 & 0.20 & 0.20 & 0.20 & 0.20 \\
$u_{2}(x 2)$ & -0.2 & -0.18 & -0.17 & -0.19 & -0.20 & -0.20 & -0.20 & -0.20 & -0.20 \\
& & $(0.10)$ & $(0.08)$ & $(0.08)$ & $(0.07)$ & $(0.07)$ & $(0.05)$ & $(0.04)$ & $(0.02)$ \\
\hline S0(1,1) & 0.6 & 0.84 & 0.81 & 0.77 & 0.71 & & & & \\
& & $(0.33)$ & $(0.33)$ & $(0.36)$ & $(0.36)$ & & & & \\
S0(2,1) & 0.45 & 0.30 & 0.29 & 0.37 & 0.44 & & & & \\
& & $(0.44)$ & $(0.42)$ & $(0.44)$ & $(0.43)$ & & & & \\
\hline \hline
\end{tabular}

Table 6: Infinite Horizon: normalization of $\boldsymbol{S}_{1}^{\boldsymbol{x}}$ (DGP: subjective beliefs)

\begin{tabular}{cccccc|cccc}
\hline \hline & \multicolumn{1}{c|}{ Estimates with Subjective beliefs } & \multicolumn{5}{c}{ Estimates with Rational Expectation } \\
\hline & TRUE & $\mathrm{N}=300$ & $\mathrm{~N}=600$ & $\mathrm{~N}=1000$ & $\mathrm{~N}=2500$ & $\mathrm{~N}=300$ & $\mathrm{~N}=600$ & $\mathrm{~N}=1000$ & $\mathrm{~N}=2500$ \\
\hline$u_{1}\left(x_{1}\right)$ & 0.1 & 0.09 & 0.09 & 0.09 & 0.10 & 0.11 & 0.11 & 0.11 & 0.11 \\
& & $(0.16)$ & $(0.11)$ & $(0.09)$ & $(0.06)$ & $(0.17)$ & $(0.12)$ & $(0.09)$ & $(0.06)$ \\
$u_{1}\left(x_{2}\right)$ & 0.2 & 0.21 & 0.19 & 0.19 & 0.19 & 0.19 & 0.18 & 0.19 & 0.19 \\
& & $(0.17)$ & $(0.13)$ & $(0.10)$ & $(0.07)$ & $(0.17)$ & $(0.13)$ & $(0.10)$ & $(0.06)$ \\
$u_{2}\left(x_{1}\right)$ & 0.2 & 0.18 & 0.19 & 0.19 & 0.19 & 0.22 & 0.22 & 0.22 & 0.22 \\
& & $(0.09)$ & $(0.08)$ & $(0.07)$ & $(0.06)$ & $(0.06)$ & $(0.04)$ & $(0.03)$ & $(0.02)$ \\
$u_{2}\left(x_{2}\right)$ & -0.2 & -0.20 & -0.20 & -0.21 & -0.21 & -0.23 & -0.22 & -0.23 & -0.23 \\
& & $(0.10)$ & $(0.09)$ & $(0.09)$ & $(0.08)$ & $(0.06)$ & $(0.05)$ & $(0.04)$ & $(0.02)$ \\
\hline S0(1,1) & 0.7 & 0.81 & 0.77 & 0.76 & 0.72 & & & & \\
& & $(0.33)$ & $(0.35)$ & $(0.34)$ & $(0.31)$ & & & & \\
S0(2,1) & 0.3 & 0.28 & 0.33 & 0.36 & 0.35 & & & & \\
& & $(0.42)$ & $(0.43)$ & $(0.43)$ & $(0.40)$ & & & & \\
\hline \hline
\end{tabular}

Table 7: Distribution of observations by number of years of employment data

\begin{tabular}{lccccccccccccccccc}
\hline \hline \# years & 6 & 7 & 8 & 9 & 10 & 11 & 12 & 13 & 14 & 15 & 16 & 17 & 18 & 19 & 20 & 21 & 22 \\
\# obs & 97 & 90 & 114 & 101 & 92 & 97 & 98 & 103 & 81 & 109 & 97 & 86 & 97 & 91 & 65 & 74 & 275 \\
cum \% & 0.05 & 0.11 & 0.17 & 0.23 & 0.28 & 0.33 & 0.39 & 0.45 & 0.49 & 0.56 & 0.61 & 0.66 & 0.71 & 0.77 & 0.80 & 0.84 & 1.00 \\
\hline \hline
\end{tabular}


Table 8: Descriptive Statistics

\begin{tabular}{lcccccc}
\hline \hline & Sample size & Mean & Std. Dev. & 5-th pctile & median & 95-th pctile \\
\hline age & 23,225 & 48.77 & 5.97 & 40 & 48 & 59 \\
education $^{\dagger}$ & 23,225 & 3.96 & 1.86 & 1 & 4 & 7 \\
annual income $(10 \mathrm{~K} \$)$ & 23,225 & 5.76 & 6.00 & 0.67 & 4.81 & 13.31 \\
employment & 23,225 & .58 & .49 & 0 & 1 & 1 \\
\hline \hline
\end{tabular}

$\dagger$ Education is classified into nine groups. 1: 0-5 grades; 2: 6-8 grades; 3: some high school; 4: completed high school; 5: 12 grades plus non-academic training; 6: college, no degree; 7: college, bachelors degree; 8: college, advanced or professional degree, some graduate work; 9: not reported.

Table 9: Estimates of Income Transitions and Preference Parameters

\begin{tabular}{|c|c|c|c|c|c|c|c|c|}
\hline & & \multicolumn{3}{|c|}{ transition $a=0$} & \multicolumn{3}{|c|}{ transition $a=1$} & \multirow{2}{*}{$\begin{array}{c}\text { preference } \\
\text { param. }\end{array}$} \\
\hline & & low & medium & high & low & medium & high & \\
\hline \multirow{6}{*}{ sub. exp. } & low & 0.394 & 0.355 & 0.252 & 0.565 & 0.283 & 0.152 & -0.096 \\
\hline & & $(0.050)$ & $(0.038)$ & $(0.019)$ & $(0.118)$ & $(0.103)$ & $(0.028)$ & $(0.122)$ \\
\hline & medium & 0.243 & 0.556 & 0.201 & 0.330 & 0.432 & 0.238 & 0.572 \\
\hline & & $(0.039)$ & $(0.064)$ & $(0.027)$ & $(0.054)$ & $(0.091)$ & $(0.038)$ & $(0.065)$ \\
\hline & high & 0.450 & 0.305 & 0.245 & - & — & - & 0.443 \\
\hline & & $(0.072)$ & $(0.043)$ & $(0.031)$ & - & - & - & $(0.163)$ \\
\hline \multirow{6}{*}{ rational exp. } & low & 0.747 & 0.250 & 0.002 & 0.752 & 0.247 & 0.002 & -0.161 \\
\hline & & $(0.015)$ & $(0.015)$ & $(0.001)$ & $(0.017)$ & $(0.016)$ & $(0.001)$ & $(0.123)$ \\
\hline & medium & 0.069 & 0.921 & 0.011 & 0.039 & 0.947 & 0.014 & 0.502 \\
\hline & & $(0.004)$ & $(0.004)$ & $(0.001)$ & $(0.002)$ & $(0.003)$ & $(0.002)$ & $(0.051)$ \\
\hline & high & 0.007 & 0.237 & 0.756 & 0.002 & 0.297 & 0.701 & 0.633 \\
\hline & & $(0.005)$ & $(0.035)$ & $(0.036)$ & $(0.002)$ & $(0.035)$ & $(0.035)$ & $(0.243)$ \\
\hline
\end{tabular}

Table 10: Simulated Conditional Choice Probabilities

\begin{tabular}{|c|c|c|c|c|c|c|c|c|c|}
\hline & \multicolumn{3}{|c|}{ sub. exp. } & \multicolumn{3}{|c|}{ rational exp. } & \multicolumn{3}{|c|}{ percentage diff. } \\
\hline & $x=1$ & $x=2$ & $x=3$ & $x=1$ & $x=2$ & $x=3$ & $x=1$ & $x=2$ & $x=3$ \\
\hline$t=55$ & 0.45885 & 0.63041 & 0.64955 & 0.47511 & 0.64550 & 0.61166 & $-3.42 \%$ & $-2.34 \%$ & $6.19 \%$ \\
\hline$t=56$ & 0.45892 & 0.63040 & 0.64931 & 0.47519 & 0.64502 & 0.61162 & $-3.42 \%$ & $-2.27 \%$ & $6.16 \%$ \\
\hline$t=57$ & 0.45913 & 0.63039 & 0.64856 & 0.47530 & 0.64431 & 0.61146 & $-3.40 \%$ & $-2.16 \%$ & $6.07 \%$ \\
\hline$t=58$ & 0.45989 & 0.63047 & 0.64612 & 0.47546 & 0.64325 & 0.61109 & $-3.27 \%$ & $-1.99 \%$ & $5.73 \%$ \\
\hline$t=59$ & 0.46286 & 0.63147 & 0.63800 & 0.47571 & 0.64165 & 0.61035 & $-2.70 \%$ & $-1.59 \%$ & $4.53 \%$ \\
\hline$t=60$ & 0.47608 & 0.63926 & 0.60894 & 0.47608 & 0.63926 & 0.60894 & $0.00 \%$ & $0.00 \%$ & $0.00 \%$ \\
\hline
\end{tabular}

